\title{
FLEXIBLE SMOOTHING AND VARIABLE SELECTION IN PARTIAL LINEAR MODELS
}

\section{TAKUMA YOSHIDA}

\author{
Graduate School of Science and Engineering \\ Kagoshima University \\ Kagoshima 890-8580 \\ Japan \\ e-mail: yoshida@sci.kagoshima-u.ac.jp
}

\begin{abstract}
This paper studies the problem of flexible estimation and variable selection in partial linear models. We estimate the parameters and smooth function in the literature of the robust regression with $M$-estimation. First, we use the radial basis function model to estimate the smooth function. For the nonlinear component, it is known that the ordinary $M$-estimation leads to obtain an over fitted curve. To overcome this, we propose the new estimation method using the sufficient dimension reduction (SDR) method. First, the SDR methods to radial basis functions are applied for trying to find small number of linear combinations of the radial bases without loss of information in the regression. Second, a multiple linear regression model between a response and the transformed radial bases is assumed and ordinary $M$-estimation is provided. As the result, using the proposed method, the variance reduction can be achieved and hence it avoids the over fitting. In the parametric component, on the other hand, we practice the variable selection using the penalization methods, such as Lasso, SCAD, and Adaptive Lasso. The theoretical justification is proved and simulation results are given. Real data examples are also addressed.
\end{abstract}

2010 Mathematics Subject Classification: 62F12, 62J02, 62 G07.

Keywords and phrases: partial linear model, sufficient dimension reduction, thin plate spline, variance reduction, variable selection.

Received October 2, 2015

(ㄷ) 2015 Scientific Advances Publishers 


\section{Introduction}

Partial linear model is a popular semiparametric model to investigate the relationship between an one-dimensional response and a multidimensional predictor. The response is explained by sum of the predictor having the parametric structure and the nonparametric structure. That is, the partial linear model is defined as following form:

$$
Y=\boldsymbol{X}^{T} \beta+g(\boldsymbol{Z})+\varepsilon,
$$

where $Y$ is a scalar response, $\boldsymbol{X}, \boldsymbol{Z}$ are $p$ and $q$ dimensional predictors, $\beta$ is an unknown parameter vector, $g$ is an unknown smooth function, and $\varepsilon$ is the error. The classical estimation methods for partial linear models have been developed by Heckman [18], Chen [6], Bhattacharya and Zhao [2], Carroll et al. [4], Xia and Härdle [41] and so on. The fundamental properties are included in Härdle et al. [15]. The partial linear model is an efficient technique not only for the mean regression but also for the quantile regression. Quantile regression estimates the conditional percentile of $Y$ given predictors and was suggested by Koenker and Bassett [22]. He and Shi [17], Lee [24], Sun [36], and Hoshino [19] studied the partial linear models in quantile regression. We consider the partial linear model for both mean regression and quantile regression. Therefore for convenience, we study the general robust regression with $M$-estimation.

For the parametric component, we play the variable selection to remove the irrelevant predictors. As the variable selection methods, several methods have been developed in the penalization framework. Notable methods include the Lasso (Tibshirani [37]), Bridge regression (Frank and Friedman [14]), SCAD (Fan and Li [13]), Adaptive Lasso (Zou [50]) and so on. Li and $\mathrm{Zhu}$ [30], Wu and Liu [40], Belloni and Chernozhukov [1], and Jiang et al. [20] developed the variable selection using above penalized methods in quantile regression. As the advantage

of SCAD and Adaptive Lasso, they have unbiasedness, sparsity and 
continuity (see, Fan and $\mathrm{Li}$ [13]) and so called oracle property (see Subsection 2.2). These properties motivate us to use the SCAD and the Adaptive Lasso.

For the nonparametric component, the function $g$ is often assumed to be an additive model since it avoids curse of dimensionality. However, the additive model is somewhat unsuitable when there is interaction between components of the predictors. Therefore in order to investigate the surface curve with interaction structure, we consider the radial basis function model: $g(\boldsymbol{z})=\sum_{k=1}^{K} u_{k} \phi_{k}(\boldsymbol{z})=\boldsymbol{u}^{T} \phi(\boldsymbol{z})$, where $\boldsymbol{u}=\left(u_{1}, \cdots, u_{K}\right)^{T}$ is an unknown parameter vector, $\phi(\boldsymbol{z})=\left(\phi_{1}(\boldsymbol{z}), \cdots, \phi_{K}(\boldsymbol{z})\right)^{T}$ is a known radial basis function vector. We can estimate $\beta$ and $\boldsymbol{u}$ by general $M$-estimation method. However, it is known that the ordinary $M$-estimation gives that the estimator of $g$ tends to have a wiggly curve when a large $K$ is used. On the other hand, for a small $K$, the estimator may not capture the surface structure.

To overcome this, Wood [39] proposed the penalization method. Unfortunately, the method of Wood [39] is applicable for only mean regression and it is not suitable for quantile regression and general robust regression. Although Koenker and Portnoy [23], Bollaerts et al. [3], Reiss and Huang [34] and other authors studied the penalized estimation of the quantile regression, it is for the univariate regression case. When $q \geq 2$, Zhu et al. [49] developed the $M$-estimation using multivariate kernel method. However, for kernel smoothing, the band-width selection is needed and the computational cost is drastically increased for $q \geq 2$. This motivates us to propose the new estimation method of the nonparametric component to obtain an estimator with smooth in $M$-estimation. Thus, the variable selection and the flexible smoothing are studied in this paper. From the mathematical point of view, the over fitting curve indicates that the estimator has a large variance and a small bias. Consequently, we propose the variance reduction estimator to obtain the flexible curve. 
To obtain the variance reduction estimator of $g$, we use the sufficient dimension reduction (SDR) method. The SDR methods are applied for trying to find small number of linear combinations of the predictors without loss of information in the regression. In the other words, for response $Y \in \mathbb{R}$ and predictor $Z \in \mathbb{R}^{q}$, the purpose of the SDR method is to find the matrix $B \in \mathbb{R}^{d \times q}$ such that

$$
Y \perp Z \mid B Z .
$$

If $d$ is smaller than $q$, the dimension reduction is succeeded. The matrix $B$ satisfying (2) and its common space spanned by each column of $B$ are called a dimension reduction subspace. The intersection of all dimension reduction subspace is so-called central dimension reduction subspace. Roughly speaking, the central dimension reduction subspace is determined as the space spanned by each column of $B$ satisfying (2) with minimum $d$. Several SDR methods have been studied, for example, sliced inverse regression (SIR) (Li [25]), sliced average variance estimation (SAVE) (Cook and Weisberg [9]), minimum average variance estimation (MAVE) (Xia et al. [42]), directional regression (DR) (Li and Wang [28]) and related methods. When the SDR method is applied to regression analysis, the model can be written as

$$
Y_{i}=f\left(\boldsymbol{Z}_{i}\right)+\varepsilon_{i}=h\left(B \boldsymbol{Z}_{i}\right)+\varepsilon_{i}
$$

where $f: \mathbb{R}^{q} \rightarrow \mathbb{R}$ and $h: \mathbb{R}^{d} \rightarrow \mathbb{R}$ are unknown functions. In this paper, we apply the SDR method to radial basis function bases. That is, we estimate the matrix $\Theta \in \mathbb{R}^{d \times K}$ such that

$$
Y-\boldsymbol{X}^{T} \beta \perp \phi(\boldsymbol{Z}) \mid \Theta \phi(\boldsymbol{Z}),
$$

using the SDR method such as SIR, SAVE and other methods. Next, consider the regression model

$$
Y_{i}-\boldsymbol{X}_{i}^{T} \beta=h\left(\Theta \phi\left(\boldsymbol{Z}_{i}\right)\right)+\varepsilon_{i}
$$


In this paper, we assume that $h$ is multiple linear model. Since $\beta$ is unknown, we use the initial estimator of $\beta$. Using the initial estimator of $\beta$, we estimate $\Theta$ and the parameters included in $h$. After estimating the nonparametric component, writing $\hat{h}(\hat{\Theta} \phi(z))$, we estimate $\beta$ with the response $Y_{i}-\hat{h}\left(\hat{\Theta} \phi\left(Z_{i}\right)\right)$ and the predictor $\boldsymbol{X}_{i}$ using SCAD and Adaptive Lasso. Thus, the proposed method is based on the one-step alternating algorithm. Yoshida [46] also studied the surface estimation with SDR method. However, they only used the SIR as SDR method whereas we focus on SIR and SAVE. Furthermore, they did not work the variable selection of the parametric component. Consequently, our method can be seen as the extended version of Yoshida [46].

This paper is organized as follows. In Section 2, we review existing the SDR method and the variable selection method with penalization. Section 3 proposes the estimation method and algorithm. In Section 4, the asymptotic results of the proposed estimator are shown. The oracle property of the estimator in parametric component is also discussed. In Section 5, we investigate to conform the performance of the estimator by simulation study. In Section 6, the real data example is addressed. We conclude the paper with Section 7. The outline of the proofs of theorems in this paper is described in Appendix.

\section{Review the Existing Methods}

In our proposed estimator, the existing SDR method and variable selection methods are used. We briefly sketch these methods in this section.

\subsection{Sufficient dimension reduction}

Let $(Y, \boldsymbol{Z}) \in \mathbb{R} \times \mathbb{R}^{q}$ be a pair of random variable, $\left\{\left(Y_{i}, \boldsymbol{Z}_{i}\right): i=1\right.$, $\cdots, n\}$ be i.i.d. random sample from $(Y, \boldsymbol{Z})$, and let $F(y \mid \boldsymbol{z})=P(Y<y \mid \boldsymbol{Z}=\boldsymbol{z})$ be a conditional distribution function of $Y$ given $\boldsymbol{Z}=\boldsymbol{z}$. The purpose of the SDR method is to find $d \times q$ matrix $B$ satisfying: 


$$
F(y \mid \boldsymbol{z})=F(y \mid B \boldsymbol{z}) .
$$

If such $B$ with $d<q$ can be found, the dimension of the predictor affecting a response $Y$ is reduced from $q$ to $d$ without loss of information. That is, the dimension reduction is succeeded. Several SDR methods can be formulated as the eigenvalue problem:

$$
M \boldsymbol{b}_{j}=\lambda_{j} G \boldsymbol{b}_{j}, \quad j=1, \cdots, q
$$

where the $q$ square matrix $M$ is symmetric and nonnegative definite, the $q$ square matrix $G$ is positive definite, vectors $\boldsymbol{b}_{1}, \cdots, \boldsymbol{b}_{q}$ are eigenvectors satisfying $\boldsymbol{b}_{i}^{T} G \boldsymbol{b}_{j}=1$ for $i=j$, and 0 otherwise, and $\lambda_{1} \geq \cdots \geq \lambda_{q} \geq 0$ are corresponding eigenvalues. Using $\boldsymbol{b}_{1}, \cdots, \boldsymbol{b}_{d}$, we define $B=\left[\boldsymbol{b}_{1}, \cdots, \boldsymbol{b}_{d}\right]^{T}$.

Specifying $M$ and $G$, several dimension reduction methods can be obtained. Let $\sum=E\left[\{\boldsymbol{Z}-E[\boldsymbol{Z}]\}^{2}\right]$ and $\boldsymbol{Z}^{*}=\Sigma^{-1}(\boldsymbol{Z}-E[\boldsymbol{Z}])$ be a covariance matrix of $\boldsymbol{Z}$ and standardized version of $\boldsymbol{Z}$. When $M=\mathrm{Cov}$ $(E[Z-E[Z] \mid y])$ and $G=\Sigma$, it is for SIR. The SAVE is defined as $M=\Sigma^{1 / 2} E\left[\left\{I-\operatorname{Cov}\left(Z^{*} \mid y\right)\right\}^{2}\right] \Sigma^{1 / 2}$ and $G=\Sigma$. The estimator of $B$ is obtained by (3) with the sample version of $M$ and $G$. Because of space limitations, we do not mention the full algorithm of SDR method here. The estimation of $B$ for SIR and SAVE have been clarified in Li [25] and Cook and Weisberg [9], respectively. In this paper, we use the SDR method by replacing $Y$ and $\boldsymbol{Z}$ to $Y-\boldsymbol{X}^{T} \beta$ and $\phi(\boldsymbol{Z})$, respectively.

Other methods are summarized in $\mathrm{Li}$ [27] and Chen et al. [7]. The minimum average variance estimation by Xia et al. [42] is also useful to obtain the estimator of $B$ although it is not in the frame of (3).

\subsection{Variable selection with penalization}

Let $(Y, \boldsymbol{X}) \in \mathbb{R} \times \mathbb{R}^{p}$ be a pair of random variable, $\left\{\left(Y_{i}, \boldsymbol{X}_{i}\right): i=1\right.$, $\cdots, n\}$ be i.i.d. random sample from $(Y, \boldsymbol{X})$. Consider the linear regression model 


$$
Y_{i}=X_{i}^{T} \beta+\varepsilon_{i}, \quad i=1, \cdots, n,
$$

where all $Y_{i}$ 's are centered and all elements of $\boldsymbol{X}_{i}$ are standardized. We then assume that some elements of $\beta$ are 0 , and hence the coefficient vector has a form $\beta=\left[\beta_{1}^{T}, \beta_{2}^{T}\right]^{T}$ with $\beta_{1} \in \mathbb{R}^{p_{1}}, p_{2}$-vector $\beta_{2}=(0, \cdots, 0)^{T}$ and $p_{1}+p_{2}=p$. We want to construct the estimator $\hat{\beta}=\left[\hat{\beta}_{1}^{T}, \hat{\beta}_{2}^{T}\right]^{T}$ of $\beta$ such that $\hat{\beta}_{1}$ has $\sqrt{n}$-consistency and $\hat{\beta}_{2}$ is exactly $\mathbf{0}$. To achieve this, the penalized method is often used. That is, $\beta$ is estimated by minimizing

$$
\sum_{i=1}^{n} \rho\left(Y_{i}-\boldsymbol{X}_{i}^{T} \beta\right)+\sum_{j=1}^{p} p_{\lambda}\left(\left|\beta_{j}\right|\right),
$$

where $\rho$ is the convex loss function, $p_{\lambda}\left(\left|\beta_{j}\right|\right)$ is the penalty to obtain $\hat{\beta}_{2}=\mathbf{0}$ and $\lambda>0$ is the smoothing parameter. Several penalties have been studied such as Lasso, bridge, SCAD, and Adaptive Lasso. The Lasso penalty is $p_{\lambda}\left(\left|\beta_{j}\right|\right)=\lambda\left|\beta_{j}\right|$, the bridge penalty is $p_{\lambda}\left(\left|\beta_{j}\right|\right)=\lambda\left|\beta_{j}\right|^{\gamma}$ for $\gamma \in(0,1)$, the SCAD penalty is given as

$$
p_{\lambda}^{\prime}\left(\left|\beta_{j}\right|\right)=\frac{d p_{\lambda}\left(\left|\beta_{j}\right|\right)}{d \beta_{j}}=\lambda\left\{I\left(\beta_{j} \leq \lambda\right)+\frac{\left(a \lambda-\beta_{j}\right)_{+}}{(a-1) \lambda} I\left(\beta_{j}>\lambda\right)\right\},
$$

where $I$ is an indicator, $(x)_{+}=\max \{0, x\}$ and $a>0$ is a constant. The Adaptive Lasso penalty is defined as $p_{\lambda}\left(\left|\beta_{j}\right|\right)=\lambda \omega_{j}\left|\beta_{j}\right|$, where $\omega_{j} \geq 0$ is a weight to control the constrain by different level for each $\beta_{j}$. As an typical choice of the weight, $\omega_{j}=1 /\left|\widetilde{\beta}_{j}\right|$ is used, where $\widetilde{\beta}_{j}$ is the initial estimator of $\beta_{j}$. In the framework of variable selection with penalization, the important properties the so called "oracle property" is often discussed. The estimator $\hat{\beta}$ has an oracle property if and only if (i) 
sparsity: with probability tending to one, $\hat{\beta}_{2}=\mathbf{0}$, and (ii) asymptotic normality: $\sqrt{n}\left(\hat{\beta}_{1}-\beta_{1}\right)$ is asymptotically distributed as normal, are satisfied. In the mean regression, Fan and Li [13] showed the oracle property of the SCAD estimator and Zou [50] studied that the estimator obtained with the Adaptive Lasso has the oracle property. In quantile regression, $\mathrm{Wu}$ and Liu [40] showed SCAD estimator and Adaptive Lasso estimator have the oracle property. Furthermore, it is known that SCAD and Adaptive Lasso have three efficient properties that unbiasedness, sparsity and continuity. These properties lead us to use the SCAD and Adaptive Lasso in the proposed method. By replacing $Y$ to $Y-\hat{g}(\boldsymbol{Z})$, these methods can directly be applied.

\section{Estimation}

Let $\left\{\left(Y_{i}, \boldsymbol{X}_{i}, \boldsymbol{Z}_{i}\right): i=1, \cdots, n\right\}$ be a random sample from the following partial linear model:

$$
Y_{i}=\mu+\boldsymbol{X}_{i}^{T} \beta+g\left(\boldsymbol{Z}_{i}\right)+\varepsilon_{i}, \quad i=1, \cdots, n,
$$

where $Y_{i} \in \mathbb{R}$ is a response, $\boldsymbol{X}_{i} \in \mathbb{R}^{p}$ and $\boldsymbol{Z}_{i} \in \mathbb{R}^{q}$ are predictors, $\mu$ is an unknown parameter, $\beta=\left(\beta_{1}, \cdots, \beta_{p}\right)^{T} \in \mathbb{R}^{p}$ is an unknown parameter vector, and $g$ is the radial basis function model:

$$
g(\boldsymbol{z})=\sum_{k=1}^{K} u_{k} \phi_{k}(\boldsymbol{z})=\boldsymbol{u}^{T} \phi(\boldsymbol{z}),
$$

$\boldsymbol{u}=\left(u_{1}, \cdots, u_{K}\right)^{T}$ is an unknown parameter vector, $\phi(\boldsymbol{z})=\left(\phi_{1}(\boldsymbol{z}), \cdots\right.$, $\left.\phi_{K}(\boldsymbol{z})\right)^{T}, \phi_{k}(\boldsymbol{x})=\phi\left(\left\|\boldsymbol{z}-\kappa_{k}\right\|\right)$ is a known radial basis function basis, $\kappa_{1}, \cdots, \kappa_{K}$ are the locations of $q$-dimensional knots, $\varepsilon_{i}$ 's are i.i.d. errors. The predictor $\boldsymbol{X}_{i}$ is standardized. Although several $\phi$ can be considered, we use the thin plate spline basis, which is of the form 


$$
\phi_{k}(\boldsymbol{z})=\phi\left(\left\|\boldsymbol{z}-\kappa_{k}\right\|\right)= \begin{cases}\left\|\boldsymbol{z}-\kappa_{k}\right\|^{2 m-q}, & q \text { odd, } \\ \left\|\boldsymbol{z}-\kappa_{k}\right\|^{2 m-q} \log \left\|\boldsymbol{z}-\kappa_{k}\right\|, & q \text { even, }\end{cases}
$$

where $m$ is an integer satisfying $2 m-q>0$ that controls the smoothness of $\phi$. If $q=2, m=2$ is often used.

We now estimate the parameter vector $\beta$ and function $g$. The ordinary estimator of $\mu, \beta$, and $\boldsymbol{u}$ are obtained as the minimizer of

$$
\sum_{i=1}^{n} \rho\left(Y_{i}-\boldsymbol{X}_{i}^{T} \beta-\mu-\phi\left(\boldsymbol{Z}_{i}\right)^{T} \boldsymbol{u}\right)
$$

where $\rho$ is a convex loss function with the unique minimizer at the origin. The minimizer of (4) is denoted by $\tilde{\mu}, \widetilde{\beta}$, and $\tilde{\boldsymbol{u}}$. The estimator $\widetilde{g}(\boldsymbol{z})=\tilde{\mu}+\tilde{\boldsymbol{u}}^{T} \phi(\boldsymbol{z})$ will have wiggly surface if $K$ is large (see Wood [39]). On the other hand, $\widetilde{g}$ may not capture the true surface when $K$ is small. However, the choice of location and number of knots is important but it is challenging.

We propose the new estimation method such that the estimator of $g$ has a curve with the smoothness. The proposed estimator is constructed by applying SDR method. Let $F\left(y-x^{T} \beta \mid z\right)$ be the conditional distribution of $Y-X^{T} \beta$ given $\boldsymbol{Z}=\boldsymbol{z}$. Using SDR method, we find the $d \times K$ matrix $\Theta$ such that

$$
F\left(y-\boldsymbol{x}^{T} \beta \mid \phi(\boldsymbol{z})\right)=F\left(y-\boldsymbol{x}^{T} \beta \mid \Theta \phi(\boldsymbol{z})\right) .
$$

When the matrix $\Theta$ satisfying (5) with $d<K$ exists, the regression model can be written as

$$
Y_{i}-\boldsymbol{X}_{i}^{T} \beta=h\left(\Theta \phi\left(\boldsymbol{Z}_{i}\right)\right)+\varepsilon_{i}
$$

where $h: \mathbb{R}^{d} \rightarrow \mathbb{R}$ is an unknown function. We now assume that $h$ has a linear form 


$$
h\left(x_{1}, \cdots, x_{d}\right)=\sum_{j=1}^{d} w_{j} x_{j}
$$

where $\boldsymbol{w}=\left(w_{1}, \cdots, w_{d}\right)^{T}$ is an unknown parameter vector. Then we write $\Theta=\left[\theta_{1}, \cdots, \theta_{d}\right]^{T}$ and have

$$
h(\Theta \phi(\boldsymbol{z}))=h\left(\theta_{1}^{T} \phi(\boldsymbol{z}), \cdots, \theta_{d}^{T} \phi(\boldsymbol{z})\right)=\sum_{j=1}^{d} w_{j}\left\{\theta_{j}^{T} \phi(\boldsymbol{z})\right\}=w^{T} \Theta \phi(\boldsymbol{z}) .
$$

Thus, to construct the estimator of $g$, the two-steps estimation is suggested. We first estimate $\Theta$ using SDR method and next $[\mu, \boldsymbol{w}]$ is estimated by minimizing $\rho$. Since $\Theta$ depends on unknown $\beta$, the initial estimate of $\beta$ is needed. Furthermore, we consider the variable selection problem for the parametric component. Consequently, we propose the following estimation algorithm:

Step 1. Construct $\widetilde{\beta}$ by minimizing (4).

Step 2. Using SDR method, obtain $\hat{\Theta}=\hat{\Theta}(\widetilde{\beta})$.

Step 3. Obtain $\hat{\mu}, \hat{\boldsymbol{w}}$ by minimizing

$$
\sum_{i=1}^{n} \rho\left(Y_{i}-\widetilde{\beta}^{T} \boldsymbol{X}_{i}-\mu-\boldsymbol{w}^{T} \hat{\Theta} \phi\left(Z_{i}\right)\right)
$$

Step 4. After centering $Y_{i}-\hat{\mu}-\hat{\boldsymbol{w}}^{T} \hat{\Theta} \phi\left(Z_{i}\right)$, estimate $\beta$ as

$$
\hat{\beta}=\underset{\beta}{\operatorname{argmin}}\left\{\sum_{i=1}^{n} \rho\left(Y_{i}-\beta^{T} \boldsymbol{X}_{i}-\hat{\mu}-\boldsymbol{w}^{T} \hat{\Theta} \phi\left(\boldsymbol{Z}_{i}\right)\right)+\lambda \sum_{j=1}^{p} p\left(\left|\beta_{j}\right|\right)\right\} .
$$

As the SDR method in Step 2, we basically use SIR and SAVE. However, other methods can also be applied. As a penalty in Step 4, we suggest to use SCAD or Adaptive Lasso since the estimator obtained by these method has an oracle property. 
Remark 1. The estimation procedure can be regarded as one-step iteration. The alternating approach such that iterate Steps 2-4 until converge $\hat{\beta}$ is also feasible. However, the iterated estimator is similar to the one-step estimator in practice although it is our empirical finding. Thus, we suggest to produce the one-step estimation.

Remark 2. In Step 2, we need to decide the dimension $d$. Zhu et al. [48] proposed an elegant method using a so-called Bayesian information criterion-type criterion (BIC-type). The BIC-type can be used in Step 2 of above algorithm and we are applying BIC-type in the simulation study of Section 5. Furthermore, we should determine the smoothing parameter $\lambda$ in Step 4. In this paper, we use the 5-fold cross-validation.

\section{Theoretical Property}

In this section, we study the asymptotic properties of $\hat{\Theta},\left[\hat{\mu}, \hat{\boldsymbol{w}}^{T}\right]^{T}$ and $\hat{\beta}$. Furthermore, we show that the variance of the proposed estimator $\hat{g}(\boldsymbol{z})$ is smaller than that of the ordinary $M$-estimator $\hat{g}(\boldsymbol{z})$. First, we give the assumption of the loss function $\rho$. For simplicity, we write $f(\boldsymbol{x}, \boldsymbol{z})=\mu+\boldsymbol{x}^{T} \beta+\boldsymbol{u}^{T} \phi(\boldsymbol{z})$. Let $C(\nu)=\operatorname{Cov}[E[\nu(Y-f(\boldsymbol{x}, \boldsymbol{z})) \mid \boldsymbol{X}, \boldsymbol{Z}]$

$\left.\left[1, \boldsymbol{X}^{T}, \boldsymbol{Z}^{T}\right]^{T}\left[1, \boldsymbol{X}^{T}, \boldsymbol{Z}^{T}\right]^{T}\right]$ for bounded function $\nu$. The following assumption is general conditions for $M$-estimation in the robust regression.

\section{Assumption 1}

(a) With probability $1, \rho(Y-f(\boldsymbol{x}, \boldsymbol{z})-t)$ and its first, second derivative and square of first derivative as a function of $t$ are bounded and continuous for the neighbourhood of 0 .

(b) $E\left[\left\{\rho(Y-f(\boldsymbol{x}, \boldsymbol{z})-t)-\rho(Y-f(\boldsymbol{x}, \boldsymbol{z}))-\rho^{\prime}(Y-f(\boldsymbol{x}, \boldsymbol{z})) t\right\}^{2}\right]=o\left(t^{2}\right)$ as $t \rightarrow 0$. 
(c) There exists $\tau>0$ such that for any $\boldsymbol{x} \in \mathbb{R}^{p}$ and $\boldsymbol{z} \in \mathbb{R}^{q}$,

$$
E\left[\mid \rho^{\prime}\left(Y-\left.f(\boldsymbol{x}, \boldsymbol{z})\right|^{2+\tau} \mid \boldsymbol{X}=\boldsymbol{x}, \boldsymbol{Z}=\boldsymbol{z}\right]<\infty\right.
$$

(d) As $n \rightarrow \infty, n^{-1} \sum_{i=1}^{n} E\left[\nu\left(Y_{i}-f(\boldsymbol{x}, \boldsymbol{z})\right]\left[1, \boldsymbol{x}_{i}^{T}, \phi\left(\boldsymbol{z}_{i}\right)^{T}\right]^{T}\left[1, \boldsymbol{x}_{i}^{T}, \phi\left(\boldsymbol{z}_{i}\right)^{T}\right]\right.$ converges to a positive definite matrix $C(\nu)$ for any bounded function $\nu$.

We first obtain the asymptotic normality of the initial estimator of $(\mu, \beta, \boldsymbol{u})$. Define

$$
\left(\mu_{0}, \beta_{0}, \boldsymbol{u}_{0}\right)=\underset{\mu, \beta, \boldsymbol{u}}{\arg \min } E[\rho(Y-f(\boldsymbol{x}, \boldsymbol{z})) \mid \boldsymbol{X}=\boldsymbol{x}, \boldsymbol{Z}=\boldsymbol{z}] .
$$

Lemma 1. Under Assumption 1, as $n \rightarrow \infty$,

$$
\sqrt{n}\left[\begin{array}{l}
\tilde{\mu}-\mu_{0} \\
\widetilde{\beta}-\beta_{0} \\
\tilde{\boldsymbol{u}}-\boldsymbol{u}_{0}
\end{array}\right] \stackrel{D}{\rightarrow} N\left(0, C\left(\left\{\rho^{\prime \prime}\right\}\right)^{-1} C\left(\left\{\rho^{\prime}\right\}^{2}\right) C\left(\left\{\rho^{\prime \prime}\right\}\right)^{-1}\right) .
$$

Next the asymptotic properties of $\hat{\Theta}$ obtained by SIR and SAVE are considered. Let $\Theta_{0}$ be the $d \times K$ matrix satisfying

$$
F\left(y-x^{T} \beta_{0} \mid \phi(z)\right)=F\left(y-x^{T} \beta_{0} \mid \Theta_{0} \phi(z)\right) .
$$

We estimate $\Theta_{0}$ using SIR and SAVE method. Define $G=\operatorname{Cov}[\phi(\boldsymbol{Z})]$ and

$$
\text { SIR: } \quad M=\operatorname{Cov}\left(E\left[\phi(Z) \mid Y-X^{T} \beta_{0}\right]\right),
$$

SAVE: $M=G^{1 / 2} E\left[\left\{I-\operatorname{Cov}\left(\phi^{*}(\boldsymbol{Z}) \mid Y-\boldsymbol{X}^{T} \beta_{0}\right)\right\}^{2}\right] G^{1 / 2}$,

where $\phi^{*}(\boldsymbol{z})=G^{-1}(\phi(\boldsymbol{Z})-E[\phi(\boldsymbol{Z})])$. Consider the eigen-equation

$$
M \theta_{j}=\lambda_{j} G \theta_{j}, \quad j=1, \cdots, K,
$$

where $\lambda_{1} \geq \cdots \geq \lambda_{K}$ are eigenvalues and $\left\{\theta_{1}, \cdots, \theta_{K}\right\}$ are corresponding eigenvectors. Then $\Theta_{0}$ is defined as $\Theta_{0}=\left[\theta_{1}, \cdots, \theta_{d}\right]^{T}$. The estimator 
$\hat{\Theta}$ of $\Theta_{0}$ is obtained by eigenvectors that are associated with the nonzero eigenvalues of (6) with the sample version of $M$ and $G$. Zhu and $\mathrm{Ng}$ [47] and $\mathrm{Li}$ and $\mathrm{Zhu}$ [29] have developed the asymptotic property of SIR and SAVE, respectively. The asymptotic consistency of $\hat{\Theta}$ in this paper can be derived in the same manner as Theorem 2 of Zhu and Ng [47] for SIR and Theorems 2.1 and 2.2 of $\mathrm{Li}$ and $\mathrm{Zhu}$ [29] for SAVE. To show the consistency of $\hat{\Theta}$, the following assumptions for SIR and SAVE are needed.

\section{Assumption 2}

(a) For any $\boldsymbol{c} \in \mathbb{R}^{K}, E\left[\boldsymbol{c}^{T} \phi(\boldsymbol{Z}) \mid \Theta \phi(\boldsymbol{Z})\right]$ is linear in $\left\{\theta_{1}^{T} \phi(\boldsymbol{Z}), \cdots\right.$, $\left.\theta_{d}^{T} \phi(\boldsymbol{Z})\right\}$

(b) For any $\boldsymbol{c} \in \mathbb{R}^{K}, V\left[\boldsymbol{c}^{T} \phi(\boldsymbol{Z}) \mid \Theta \phi(\boldsymbol{Z})\right]$ is constant.

(c) For any $s \in \mathbb{R}$, each component of $K$-vector $m(s)=E[\phi(\boldsymbol{Z}) \mid Y-$ $\left.\boldsymbol{X}^{T} \beta_{0}=s\right]$ and $K$-square matrix $M(s)=\operatorname{Cov}\left(\phi^{*}(\boldsymbol{Z}) \mid Y-\boldsymbol{X}^{T} \beta_{0}=s\right)$ has the continuous first order differential.

(d1) For SIR, with a response $Y-X^{T} \beta_{0}$ and a predictor $\phi^{*}(Z)$, conditions (i)-(iv) of Theorem 2 of Zhu and $\mathrm{Ng}$ [47] hold.

(d2) For SAVE, with a response $Y-\boldsymbol{X}^{T} \beta_{0}$ and a predictor $\phi^{*}(\boldsymbol{Z})$, conditions (2), (3) of Theorem 2.1 and (1)-(6) of Theorem 2.2 of $\mathrm{Li}$ and Zhu [29] hold.

For only next lemma, we denote $\Theta_{0}$ for SIR and SAVE as $\Theta_{0, S I R}$ and $\Theta_{0, S A V E}$ and these estimators are labelled as $\hat{\Theta}_{S I R}$ and $\hat{\Theta}_{S A V E}$.

Lemma 2. Under Assumptions 1-2, as $n \rightarrow \infty$, for $S I R, n^{1 / 2}\left(\hat{\Theta}_{S I R}-\right.$ $\left.\Theta_{0, S I R}\right)=O_{P}(1)$ and for $S A V E$, there exists $0<\beta<1 / 2$ such that $n^{\beta}\left(\hat{\Theta}_{S A V E}-\Theta_{0, S A V E}\right)=O_{P}(1)$. 
By Lemmas 1 and 2, the asymptotic normality of the $(\hat{\mu}, \hat{\boldsymbol{w}})$ is obtained. Let $\left(\mu_{0}, \boldsymbol{w}_{0}\right)$ be the minimizer of

$$
E\left[\rho\left(Y-\boldsymbol{x}^{T} \beta_{0}-\mu-\boldsymbol{w}^{T} \Theta_{0} \phi(\boldsymbol{z})\right) \mid \boldsymbol{X}=\boldsymbol{x}, \boldsymbol{Z}=\boldsymbol{z}\right] .
$$

Let

$$
\Sigma_{\phi}(\nu)=\operatorname{Cov}\left\{\sqrt{E[\nu(Y-f(\boldsymbol{x}, \boldsymbol{z})) \mid \boldsymbol{X}=\boldsymbol{x}, \boldsymbol{Z}=z]}\left[1\left\{\Theta_{0} \phi(\boldsymbol{Z})\right\}^{T}\right]^{T}\right\} .
$$

Theorem 1. Suppose that the matrix $\Sigma_{\phi}(v)$ is positive definite for any bounded function $\nu$. Under the Assumptions 1-2, as $n \rightarrow \infty$,

$$
\sqrt{n}\left[\begin{array}{l}
\hat{\mu}-\mu_{0} \\
\hat{\boldsymbol{w}}-\boldsymbol{w}_{0}
\end{array}\right] \stackrel{D}{\rightarrow} N\left(0, \Sigma_{\phi}\left(\rho^{\prime \prime}\right)^{-1} \Sigma_{\phi}\left(\left\{\rho^{\prime}\right\}^{2}\right) \Sigma_{\phi}\left(\rho^{\prime \prime}\right)^{-1}\right) .
$$

Lastly, we show the asymptotic properties of $\hat{\beta}$. We write the SCAD penalty and the Adaptive Lasso penalty as $p_{\lambda, S C}\left(\left|\beta_{j}\right|\right)$ and $p_{\lambda, A L}\left(\left|\beta_{j}\right|\right)$, respectively. Furthermore as the weight $w_{j}$ for $p_{\lambda, A L}\left(\left|\beta_{j}\right|\right)$, we use $\widetilde{w}_{j}=1 /\left|\beta_{j}\right|$. Let $\beta_{0}$ be the minimizer of

$$
E\left[\rho\left(Y-\boldsymbol{x}^{T} \beta-\mu_{0}-\boldsymbol{w}_{0}^{T} \Theta_{0} \phi(\boldsymbol{z})\right) \mid \boldsymbol{X}=\boldsymbol{x}, \boldsymbol{Z}=\boldsymbol{z}\right] .
$$

We assume that the parameter vector $\beta_{0}$ in (7) can be written as $\beta_{0}=\left[\beta_{01}^{T}, \beta_{02}^{T}\right]^{T} \quad$ with $\quad p_{1}$-vector $\beta_{01} \in \mathbb{R}^{p_{1}}$ and $p_{2}$-vector $\beta_{02}=\mathbf{0}$, where $p_{1}+p_{2}=p$. Define $C_{01}(\nu)=\operatorname{Cov}\left[E[v(Y-f(\boldsymbol{x}, \boldsymbol{z})) \mid \boldsymbol{X}, \boldsymbol{Z}] \boldsymbol{X}_{1}\right]$ for bounded function $\nu$, where $\boldsymbol{X}_{1}$ is first $p_{1}$-vector of $\boldsymbol{X}$.

Corresponding to $\beta_{0}$, the estimator can be partitioned as $\hat{\beta}=\left[\hat{\beta}_{1}^{T}, \hat{\beta}_{2}^{T}\right]^{T}$. The following theorems are the oracle property of the $\hat{\beta}$ for the SCAD penalty and the Adaptive Lasso penalty. 
Theorem 2. Under Assumptions 1-2, using $p_{\lambda, S C}\left(\left|\beta_{j}\right|\right)$ as the penalty, $\lambda \rightarrow 0$ and $\sqrt{n} \lambda \rightarrow \infty$ as $n \rightarrow \infty$,

(i) Sparsity : with probability tending to one, $\hat{\beta}_{2}=\mathbf{0}$.

(ii) Asymptotic normality : $\sqrt{n}\left(\hat{\beta}_{1}-\beta_{01}\right) \stackrel{D}{\rightarrow} N\left(\mathbf{0}, C_{01}\left(\left\{\rho^{\prime \prime}\right\}\right)^{-1} C_{01}\left(\left\{\rho^{\prime}\right\}^{2}\right)\right.$ $\left.C_{01}\left(\left\{\rho^{\prime \prime}\right\}\right)^{-1}\right)$.

Theorem 3. Under Assumptions 1-2, using $p_{\lambda, A L}\left(\left|\beta_{j}\right|\right)$ as the penalty, $n \lambda \rightarrow \infty$ and $\sqrt{n} \lambda \rightarrow 0$ as $n \rightarrow \infty$,

(i) Sparsity : with probability tending to one, $\hat{\beta}_{2}=\mathbf{0}$.

(ii) Asymptotic normality : $\sqrt{n}\left(\hat{\beta}_{1}-\beta_{01}\right) \stackrel{D}{\rightarrow} N\left(\mathbf{0}, C_{01}\left(\left\{\rho^{\prime \prime}\right\}\right)^{-1} C_{01}\left(\left\{\rho^{\prime}\right\}^{2}\right)\right.$ $\left.C_{01}\left(\left\{\rho^{\prime \prime}\right\}\right)^{-1}\right)$.

We now discuss about the variance of the estimator $\hat{g}(\boldsymbol{z})$. Since $\hat{\theta}_{1}, \cdots, \hat{\theta}_{d}$ are obtained from the SDR matrix (see Subsection 2.1), for $i \neq j$, the inner product of $\hat{\theta}_{i}$ and $\hat{\theta}_{j}$ is 0 . That is, we have $\phi(\boldsymbol{z})^{T} \hat{\theta}_{i} \hat{\theta}_{j}^{T} \phi(\boldsymbol{z})=0$. Thus, the expectation of the square of $\hat{g}(\boldsymbol{z})$ can be written as

$$
E\left[\hat{g}(\boldsymbol{z})^{2}\right]=\sum_{j=1}^{d} E\left[\hat{w}_{j}^{2}\left\{\hat{\theta}_{j}^{T} \phi(\boldsymbol{z})\right\}^{2}\right] .
$$

On the other hand, from Theorem 1, the expectation of $\hat{g}(\boldsymbol{z})$ can be asymptotically expressed as

$$
E[\hat{g}(\boldsymbol{z})]=\sum_{j=1}^{d} w_{0_{j}} \theta_{0_{j}}^{T} \phi(\boldsymbol{z})+o(1) .
$$


For $i \neq j$, we also obtain $\phi(\boldsymbol{z})^{T} \theta_{0 i} \theta_{0_{j}}^{T} \phi(\boldsymbol{z})=0$ and hence the asymptotic variance of $\hat{g}(\boldsymbol{z})$ is

$$
\begin{aligned}
V[\hat{g}(\boldsymbol{z})] & =\sum_{j=1}^{d}\left\{E\left[\hat{w}_{j}^{2}\left\{\hat{\theta}_{j}^{T} \phi(\boldsymbol{z})\right\}^{2}\right]-w_{0_{j}}^{2}\left\{\theta_{0_{j}}^{T} \phi(z)\right\}^{2}\right\}+o(1) \\
& =\sum_{j=1}^{d} V\left[\hat{w}_{j}^{2}\left\{\theta_{j}^{T} \phi(\boldsymbol{z})\right\}^{2}\right]+o(1) .
\end{aligned}
$$

Since $V\left[\hat{w}_{j}^{2}\left\{\hat{\theta}_{j}^{T} \phi(\boldsymbol{z})\right\}^{2}\right] \geq 0$ for $j=1, \cdots, d$, the dimension $d$ controls the variance of the nonlinear estimator $\hat{g}(\boldsymbol{z})$. When $d=K$, the proposed estimator is reduced to the ordinary $M$-estimator $\tilde{\boldsymbol{u}}^{T} \phi(\boldsymbol{z})$. That is, $V[\hat{g}(\boldsymbol{z})]=\sum_{j=1}^{K} V\left[\hat{w}_{j}^{2}\left\{\hat{\theta}_{j}^{T} \phi(\boldsymbol{z})\right\}^{2}\right]+o(1)$. It indicates that the proposed method constructs the variance reduction estimator rather than the ordinary estimator. From Theorems 2 and 3, the accuracy of the variable selection of parametric component is guaranteed. Furthermore, in the nonlinear component, the variance reduction estimation can be achieved using SDR method.

Remark 3. We first note that Assumption 2(b) is not needed for SIR. When SAVE is used, both Assumption 2(a) and (b) are needed. Assumption 2(a) and (b) are very important to use SIR and SAVE. If these are failed, SIR and SAVE will not be included in the dimension reduction subspace and the efficient performances of SIR and SAVE are not guaranteed. In real data analysis, however, it is very difficult to conform whether $\phi(\boldsymbol{Z})$ satisfies Assumption 2(a) and (b). However, we can give some examination about this. Diaconis and Freedman [11] showed that the distribution of $\phi(\boldsymbol{Z})$ is elliptically symmetric, then Assumption 2(a) and (b) holds. Furthermore, they discussed that all lowdimensional projections of high-dimensional data are approximately 
normal. In radial basis function models, we usually choose a large $K$ to capture the smooth surface function and hence the dimension of $\phi(\boldsymbol{Z})$ is large. Thus, it appears that $\phi(\boldsymbol{Z})$ satisfies Assumption 2(a) and (b) naturally in this sense.

Remark 4. We focused on SIR and SAVE as the SDR method. In general, SAVE is more comprehensive rather than SIR. That is, the subspace spanned by $\Theta_{0}$ obtained by SAVE covers that by SIR (see, Cook and Critchley [8] and Ye and Weiss [44]). Thus, it seems that SAVE is more efficient than SIR. However, SAVE does not have $\sqrt{n}$-consisteng unless a bias correction is performed, whereas SIR has $\sqrt{n}$-consistency. In this sense, SAVE is not outperformed compared with SIR. From practical point of view, it is known that SAVE is not efficient in estimating monotone function of the argument transformed predictor $\Theta \phi(\boldsymbol{Z})$. On the other hand, if the regression surface is symmetric with respect to $\Theta \phi(\boldsymbol{Z})$, SIR fail to work. Thus, both SIR and SAVE have advantages and disadvantages with each other. Roughly speaking, if the performance of SIR is worse, SAVE then will have a good behaviour. The reverse is also true. Consequently, SIR and SAVE can be regarded as complementary methods. Actually, the estimator with SIR did not work well in the simulation of Section 5 unlike SAVE. In application to the Boston housing data of Section 6, on the other hand, the performance of SAVE is not good unlike SIR.

\section{Simulation}

In this section, we conform the performance of the proposed estimator. We focus on the mean regression and the median regression. The data $\left\{\left(y_{i}, \boldsymbol{x}_{i}, \boldsymbol{z}_{i}\right): i=1, \cdots, n\right\}$ was generated from the following partial linear models:

$$
y_{i}=\boldsymbol{x}_{i}^{T} \beta+g\left(\boldsymbol{z}_{i}\right)+\varepsilon_{i}, \quad i=1, \cdots, n,
$$

where $\boldsymbol{x}_{1}, \cdots, \boldsymbol{x}_{n} \stackrel{\text { i.i.d. }}{\sim} N_{8}(\mathbf{0}, \Sigma), \Sigma=\left(0.5^{|i-j|}\right)_{i j}, \beta=(3,1.5,0,0,2,0,0,0)^{T}$, 
Model $1: g\left(z_{1}, z_{2}\right)=\sin \left(2 \pi\left(z_{1}-0.5\right)^{2}\right) \cos \left(4 \pi z_{2}\right)$,

Model $2: g\left(z_{1}, z_{2}\right)=\exp \left[10\left\{-\left(z_{1}-0.25\right)^{2}-\left(z_{2}-0.25\right)^{2}\right\}\right]$

$$
+0.5 \exp \left[14\left\{-\left(z_{1}-0.7\right)^{2}-\left(z_{2}-0.7\right)^{2}\right\}\right]
$$

$\boldsymbol{z}_{i}=\left(z_{i 1}, z_{i 2}\right)$ was generated uniformly on $[0,1]^{2}$, the error was distributed as $N(0,1)$ and Cauchy with location 0 and scale 1 . Then $\boldsymbol{x}_{i}, \boldsymbol{z}_{i}, \varepsilon_{i}$ are independent and $\boldsymbol{x}_{i}$ is standardized. In multiple linear regression, the above model of the parametric component has been considered by many authors such as Tibshirani [37], Fan and Li [13], Zou [50], Wu and Liu [40], to name a few. The above nonparametric functions were used in Xiao et al. [43] and Wood [39], respectively. First, we showed the sample MISE of the surface estimator, which is defined as

$$
\operatorname{MISE}=\frac{1}{500} \sum_{r=1}^{500} \frac{1}{N} \sum_{i=1}^{N}\left\{\hat{g}_{r}\left(z_{i 1}^{*}, z_{i 2}^{*}\right)-g\left(z_{i 1}^{*}, z_{i 2}^{*}\right)\right\}^{2},
$$

where $\hat{g}_{r}$ is the estimator of $g$ for the $r$-th repetition and $\left\{\left(z_{i 1}^{*}, z_{i 2}^{*}\right): i=\right.$ $1, \cdots, N\}$ is the $N=50 \times 50$ regular grid on the unit square. Next, the prediction error of the estimator was evaluated. The prediction error (PE) is defined as

$$
\mathrm{PE}=\frac{1}{N} \sum_{i=1}^{N} \rho\left(y_{i, t e s t}-\boldsymbol{x}_{i, t e s t}^{T} \hat{\beta}-\hat{g}\left(\boldsymbol{z}_{i, t e s t}\right)\right),
$$

where $\left\{\left(y_{i, t e s t}, \boldsymbol{x}_{i, t e s t}, \boldsymbol{z}_{i, \text { test }}\right): i=1, \cdots, N\right\}$ is the test data with size $N$, which was generated from (8) and was not used to estimate $\beta$ and $g$. For mean and median regression, $\rho(u)=u^{2}$ and $\rho(u)=|u|$ are used, respectively. Furthermore, we counted $C$; the average number of nonzero coefficients correctly estimated to be nonzero and IC; the average number of zero coefficients incorrectly estimated to be nonzero. The sample sizes 
$n=60,90$, and 120 were used. In all simulations, we used Model 1 and Model 2 and normal error in mean regression. On the other hand, in median regression, Model 1 and normal error and Cauchy error were used.

To estimate $g$, we used the thin plate spline basis with the 4,6 , and 8 equidistant knots for the $z_{1}$-axis and $z_{2}$-axis, leading to a total number of knots $K=16,36$, and 64 . For $n=60, K=64$ was omitted since it is abuse. As the SDR method, we used the SAVE. For the dimension of SAVE, we considered $d=1, d=K / 2, d=K$ and $d$ selected by BICtype. When $d=K$, the estimator is reduced to the initial estimator $\tilde{g}(\boldsymbol{z})$. In the estimation and variable selection for the parametric component, we used SCAD and Adaptive Lasso (AL). We will mention the result for only the case that $\hat{g}$ with $K=36$ and $d$ selected via BIC-type criterion. For comparison, we also considered the PE, C, and IC without SDR-method, which is equal to the initial estimator $\tilde{g}(\boldsymbol{z})$. Furthermore, we constructed the PE of the estimator without variable selection and with correct model, which are denoted as ordinary and oracle. Thus, we compared the estimator with SDR and with SCAD, AL, ordinary and oracle, and without SDR and with SCAD, AL, ordinary and oracle, which are labelled as SDR-SCAD, SDR-AL, SDR-ordinary, SDR-oracle, INISCAD, INI-AL, INI-ordianry, and INI-oracle.

Table 1 shows that the sample MISE of the estimator of surface function $g\left(z_{1}, z_{2}\right)$ in mean regression. For each sample size and model, the efficiency of SDR method could be found. The MISEs of the estimator with BIC were similar to those with $d=1$. It indicates that BIC selects $d=1$ or small $d$ in each replication. Compared with $d=K$, which is the initial estimator, the performance of the estimator with $d=1$ was dramatically improved. It appears that the estimator without SDR method has an over fitted curve and the proposed method leads to obtain a curve with smoothness. When $K$ is large, the reduction of MISE was remarkable. It is not surprising result since large $K$ brings to a wiggly estimate. Even with $n=120$ and $K=16$, the proposed estimator had 
better behavior than the initial estimator $(d=K)$. Thus, the behaviour of the ordinary method is quite unstable and hence the SDR method is useful even if $K$ is not too large. In this simulation, for all $K$, MISE of the estimator with $d=1$ or $d$ selected via BIC was similar. Therefore, it seems that the proposed estimator is not sensitive to the choice of $K$.

Table 1. MISEs of estimator of nonparametric function in mean regression. $K$ is the number of basis and $d$ is the dimension for SAVE. All entries for MISE are $10^{2}$ times their actual values for ease of presentation

\begin{tabular}{lccccccc}
\hline \multicolumn{3}{c}{ Model 1} & & & \multicolumn{3}{c}{ Model 2} \\
\hline Method & $K=16$ & $K=36$ & $K=64$ & & $K=16$ & $K=36$ & $K=64$ \\
\hline BIC & & & & $n=60$ & & & \\
$d=1$ & 2.124 & 2.087 & - & & 15.43 & 14.96 & - \\
$d=K / 2$ & 31.13 & 29.12 & - & & 21.25 & 46.96 & - \\
$d=K$ (INI) & 77.98 & 66.48 & - & & 46.98 & 86.53 & - \\
& & & & $n=90$ & & & \\
BIC & 1.953 & 1.873 & 1.931 & & 15.43 & 14.96 & - \\
$d=1$ & 1.973 & 1.872 & 1.931 & & 13.22 & 13.15 & 13.21 \\
$d=K / 2$ & 23.84 & 18.42 & 83.62 & & 15.99 & 27.42 & 77.56 \\
$d=K$ (INI) & 76.98 & 78.14 & 163.2 & & 23.74 & 78.01 & 125.6 \\
& & & & $n=120$ & & & \\
BIC & 1.641 & 1.251 & 1.684 & & 9.848 & 9.611 & 9.521 \\
$d=1$ & 1.703 & 1.360 & 1.688 & & 9.848 & 9.621 & 9.524 \\
$d=K / 2$ & 5.287 & 10.09 & 9.367 & & 11.42 & 13.49 & 16.76 \\
$d=K$ (INI) & 14.88 & 38.19 & 99.52 & & 14.73 & 38.07 & 99.41 \\
\hline
\end{tabular}

In Table 2, the PE, C, and IC of each estimator are illustrated. First, the PE with SDR method was quite smaller than that without SDR method. From this, the proposed method is efficient from the prediction error point of view. Although the difference is little, PE of SDRAL was smaller than SDR-SCAD. C and IC of SDR-SCAD and SDR-AL are near to 3 and 5, respectively. Especially in all replications, AL yielded that $\mathrm{C}$ is 3 even for $n=60$. 
Table 2. Resilts for predictions in mean regression

\begin{tabular}{|c|c|c|c|c|c|c|}
\hline \multirow[b]{3}{*}{ Method } & \multicolumn{3}{|c|}{ Model 1} & \multicolumn{3}{|c|}{ Model 2} \\
\hline & \multirow[b]{2}{*}{$\mathrm{PE}$} & \multicolumn{2}{|c|}{ No. of Zeros } & \multirow[b]{2}{*}{$\mathrm{PE}$} & \multicolumn{2}{|c|}{ No. of Zeros } \\
\hline & & $\mathrm{C}$ & IC & & $\mathrm{C}$ & IC \\
\hline \multicolumn{7}{|l|}{$n=60$} \\
\hline SDR-SCAD & 1.665 & 2.79 & 3.06 & 1.653 & 2.91 & 3.86 \\
\hline SDR-AL & 1.259 & 3.00 & 4.13 & 1.214 & 3.00 & 4.55 \\
\hline SDR-Ordinary & 2.500 & - & - & 2.514 & - & - \\
\hline SDR-Oracle & 1.245 & - & - & 1.134 & - & - \\
\hline INI-SCAD & 16.04 & 2.88 & 2.35 & 13.13 & 2.86 & 3.35 \\
\hline INI-AL & 14.65 & 2.93 & 1.99 & 13.35 & 2.90 & 2.01 \\
\hline INI-Ordinary & 16.56 & - & - & 16.55 & - & - \\
\hline INI-Oracle & 11.42 & - & - & 13.01 & - & - \\
\hline \multicolumn{7}{|l|}{$n=90$} \\
\hline SDR-SCAD & 1.474 & 2.48 & 3.64 & 1.113 & 3.00 & 4.69 \\
\hline SDR-AL & 0.998 & 3.00 & 4.76 & 1.106 & 3.00 & 4.76 \\
\hline SDR-Ordinary & 1.406 & - & - & 1.521 & - & - \\
\hline SDR-Oracle & 0.945 & - & - & 1.103 & - & - \\
\hline INI-SCAD & 4.233 & 3.00 & 2.94 & 4.943 & 3.00 & 4.26 \\
\hline INI-AL & 3.985 & 3.00 & 3.72 & 3.950 & 3.00 & 3.80 \\
\hline INI-Ordinary & 4.417 & - & - & 4.421 & - & - \\
\hline INI-Oracle & 3.734 & - & - & 3.731 & - & - \\
\hline \multicolumn{7}{|l|}{$n=120$} \\
\hline SDR-SCAD & 0.970 & 3.00 & 4.45 & 1.091 & 3.00 & 4.51 \\
\hline SDR-AL & 0.926 & 3.00 & 4.93 & 1.053 & 3.00 & 4.94 \\
\hline SDR-Ordinary & 1.138 & - & - & 1.285 & - & - \\
\hline SDR-Oracle & 0.914 & - & - & 0.946 & - & - \\
\hline INI-SCAD & 2.539 & 3.00 & 3.47 & 2.541 & 3.00 & 3.23 \\
\hline INI-AL & 2.363 & 3.00 & 4.33 & 2.381 & 3.00 & 4.31 \\
\hline INI-Ordinary & 2.692 & - & - & 2.695 & - & - \\
\hline INI-Oracle & 2.136 & - & - & 2.124 & - & - \\
\hline
\end{tabular}


Table 3 describes the MISE of $\hat{g}\left(z_{1}, z_{2}\right)$ in median regression. Similar to the mean regression, the performance of the proposed estimator was better than that of the ordinary estimator. The robustness of the quantile regression could also be found from the result for Cauchy error. However when $n=90$ and $K=64$, the MISE of the proposed estimator was too large and the estimator would have an unstable curve. It indicates that for data having heavier tail density, too large $K$ should not be used and so the choice of $K$ is somewhat important. In this simulation, $K=36$ is one of good choice.

Table 3. MISEs of estimator of nonparametric function in median regression. $K$ is the number of basis and $d$ is the dimension for SAVE. > 1000 means over 1000. All entries for MSE and MISE are $10^{2}$ times their actual values for ease of presentation

\begin{tabular}{lccccccc}
\hline \multicolumn{3}{c}{ Normal Error } & \multicolumn{3}{c}{ Cauchy Error } \\
\hline Method & $K=16$ & $K=36$ & $K=64$ & & $K=16$ & $K=36$ & $K=64$ \\
\hline BIC & 2.541 & 2.622 & - & & 4.149 & 9.986 & - \\
$d=1$ & 2.541 & 2.632 & - & & 4.149 & 9.986 & - \\
$d=K / 2$ & 33.79 & 47.39 & - & & 138.9 & 182.7 & - \\
$d=K$ (INI) & 97.93 & 123.3 & - & & 506.0 & 883.6 & - \\
& & & & $n=90$ & & & \\
BIC & 0.765 & 0.469 & 0.812 & & 2.651 & 1.205 & 462.8 \\
$d=1$ & 0.766 & 0.469 & 0.813 & & 2.651 & 1.205 & 501.8 \\
$d=K / 2$ & 15.51 & 26.87 & 103.24 & & 46.57 & 38.31 & $>1000$ \\
$d=K(\mathrm{INI})$ & 56.80 & 71.13 & 503.02 & & 213.9 & 584.60 & $>1000$ \\
& & & & $n=120$ & & & \\
BIC & 0.685 & 0.462 & 0.582 & & 1.490 & 0.646 & 4.278 \\
$d=1$ & 0.685 & 0.462 & 0.595 & & 1.490 & 0.646 & 4.278 \\
$d=K / 2$ & 5.659 & 19.44 & 96.90 & & 22.43 & 22.36 & 333.4 \\
$d=K(\mathrm{INI})$ & 15.59 & 45.73 & 113.06 & & 57.04 & 102.36 & $>1000$ \\
\hline
\end{tabular}


In Table 4, the results of variable selection for median regression are shown. PE of the proposed estimator was smaller than that of the ordinary estimator. For $n=60$ of Cauchy error, PE of SDR-ordinary was quite larger than other estimator with SDR method. For both SCAD and $\mathrm{AL}, \mathrm{C}$ is 3 even when $n=60$ and the initial estimator. Concerned with IC, the performance of the proposed estimator was somewhat better than that of the ordinary estimator.

Table 4. Resilts for predictions in median regression

\begin{tabular}{|c|c|c|c|c|c|c|}
\hline \multirow[b]{3}{*}{ Method } & \multirow[b]{3}{*}{$\mathrm{PE}$} & \multicolumn{2}{|c|}{ Normal Error } & \multirow[b]{3}{*}{$\mathrm{PE}$} & \multirow{2}{*}{\multicolumn{2}{|c|}{$\frac{\text { Cauchy Error }}{\text { No. of Zeros }}$}} \\
\hline & & \multicolumn{2}{|c|}{ No. of Zeros } & & & \\
\hline & & $\mathrm{C}$ & IC & & $\mathrm{C}$ & IC \\
\hline \multicolumn{7}{|l|}{$n=60$} \\
\hline SDR-SCAD & 1.044 & 3.00 & 3.53 & 7.457 & 3.00 & 4.94 \\
\hline SDR-AL & 1.027 & 3.00 & 3.33 & 7.316 & 3.00 & 4.27 \\
\hline SDR-Ordinary & 1.251 & - & - & 14.71 & - & - \\
\hline SDR-Oracle & 1.013 & - & - & 7.231 & - & - \\
\hline INI-SCAD & 9.577 & 3.00 & 3.51 & 18.39 & 3.00 & 4.15 \\
\hline INI-AL & 9.570 & 3.00 & 3.69 & 18.35 & 3.00 & 4.09 \\
\hline INI-Ordinary & 11.75 & - & - & 28.67 & - & - \\
\hline INI-Oracle & 9.452 & - & - & 15.35 & - & - \\
\hline \multicolumn{7}{|l|}{$n=90$} \\
\hline SDR-SCAD & 0.947 & 3.00 & 4.52 & 4.708 & 3.00 & 4.81 \\
\hline SDR-AL & 0.825 & 3.00 & 4.14 & 4.606 & 3.00 & 4.23 \\
\hline SDR-Ordinary & 0.848 & - & - & 8.959 & - & - \\
\hline SDR-Oracle & 0.842 & - & - & 4.601 & - & - \\
\hline INI-SCAD & 2.236 & 3.00 & 3.23 & 8.416 & 3.00 & 4.10 \\
\hline INI-AL & 2.156 & 3.00 & 3.65 & 8.327 & 3.00 & 4.09 \\
\hline INI-Ordinary & 2.195 & - & - & 10.66 & - & - \\
\hline INI-Oracle & 2.141 & - & - & 8.315 & - & - \\
\hline \multicolumn{7}{|l|}{$n=120$} \\
\hline SDR-SCAD & 0.851 & 3.00 & 4.43 & 4.576 & 3.00 & 4.60 \\
\hline
\end{tabular}


Table 4. (Continued)

\begin{tabular}{lcccccc}
\hline SDR-AL & 0.804 & 3.00 & 4.37 & 4.481 & 3.00 & 4.21 \\
SDR-Ordinary & 0.823 & - & - & 5.866 & - & - \\
SDR-Oracle & 0.801 & - & - & 4.425 & - & - \\
INI-SCAD & 2.412 & 3.00 & 4.03 & 6.313 & 3.00 & 4.25 \\
INI-AL & 2.385 & 3.00 & 3.39 & 6.243 & 3.00 & 3.96 \\
INI-Ordinary & 2.513 & - & - & 7.608 & - & - \\
INI-Oracle & 2.031 & - & - & 6.242 & - & - \\
\hline
\end{tabular}

The prediction error, $\mathrm{C}$ and IC for $K=4$ and $K=8$ were practiced although it was not described here. As the result, efficiency of the proposed method could be confirmed. The same simulation studies of median regression for Model 2 were explored. Although the results are not reported owing to lack of space, the estimators behaved well.

Remark 5. In this simulation study, we were confirmed that the performance of the proposed estimator is better than that of the ordinary $M$-estimator, which is the case $d=K$ (INI). Although we were not described the results for want of space, we compared the proposed method with the following models and methods: The partial linear additive model

$$
g(\boldsymbol{z})=g_{1}\left(z_{1}\right)+g_{2}\left(z_{2}\right)
$$

where $g_{1}$ and $g_{2}$ are univariate functions (Liu et al. [31]), the partial linear single index model

$$
g(\boldsymbol{z})=g_{0}\left(\theta^{T} \boldsymbol{z}\right)
$$

where $g_{0}$ is univariate function and $\theta$ is two dimensional vector (see Carroll et al. [4]) and the ordinary two dimensional kernel smoothing method (see Zhu et al. [49]). We also calculated the sample MISE and PE of the estimators obtained from above methods in all settings of this section. We then use the cubic $B$-spline method with 5 equidistant knots to estimate $g_{1}, g_{2}$, and $g_{0}$ for he partial linear additive model and the 
single index model, and Epanechnikov kernel with the band-width selected by the generalized cross validation for bivariate kernel method. As the result, the proposed method was superior or nearly equal than the above estimators. Although the additive models avoid the curse of dimensionality, the interaction structure of covariates can not be captured. The single index model is useful method to dimension reduction in practice. However in generally, the loss of information may be occurred since we $q$-dimensional predictor is compulsory transformed to 1 dimension. The kernel method is traditional nonparametric smoothing but the computational cost of the bandwidth selection grows when $q \geq 2$. Thus, the proposed method covers the disadvantage of the above methods. Although we can not compare the proposed method with the above and other nonparametric/nonlinear methods directly, we believe that the proposed method is one of efficient methods in partial linear models.

\section{Data Example}

We apply the proposed method to Boston housing data, which was originally analyzed by Harrison and Rubinfeld [16]. The data consists of 14 variables and $n=506$ samples. The purpose was to evaluate the effect of various predictor variables on housing price. In our model, the response is taken to be the logarithm of median value of owner occupied homes and the partial linear model is assumed. The predictors $\boldsymbol{x}=\left(x_{1}, \cdots, x_{11}\right)$ in the parametric component are $x_{1}$ : the crime rate by town; $x_{2}$ : the percentage of the town' residential land zoned for lots greater than 25,000 square feet; $x_{3}$ : the percentage of nonmetal business acres per town; $x_{4}$ : the indicator of whether the census tract borders the Charles Rivers; $x_{5}$ : nitrogen oxide concentration in parts per hundred million (pphm); $x_{6}$ : the weighted distance to five Boston employment centers from houses; $x_{7}$ : the percentage of owner units built prior to 
1940; $x_{8}$ : an index of accessibility to radial highways; $x_{9}$ : the full tax rate of the property; $x_{10}$ : the pupil-teacher ratio by town school district and $x_{11}$ : the proportion of the population that is African-American. For the nonparametric component, we consider the surface of $\boldsymbol{z}=\left(z_{1}, z_{2}\right)$, where $z_{1}$ is the average number of rooms in owner units and $z_{2}$ is the percentage of the population in the area having low economic status. The same $\boldsymbol{z}$ was used by Doksum and Koo [12] in the nonparametric smoothing.

Our purpose is to predict the conditional mean and median surface of $Y$ given above predictors. We used the thin plate spline basis and the number of knots was $K=36$ and the location was an $6 \times 6$ regular grid on the range of $\left(z_{1}, z_{2}\right)$. As the SDR method, we used SIR. The dimension of the SIR is fixed at $d=1$ to observe the linear regression between the partial residual and the transformed predictor. The Step 3 of the proposed estimation algorithm constructs the linear estimator of the partial residual and the transformed predictor by SDR method. In Figure 1, the partial residual $y_{i}-\boldsymbol{x}^{T} \tilde{\beta}$ versus $\hat{\theta}_{1}^{T} \phi\left(\boldsymbol{z}_{i}\right)$ and its linear estimator (solid line) are illustrated. The left panel is for mean regression and the right panel is for median regression. For both panels, we see that the linear estimator can captures the mean and median structure of the partial residual. 

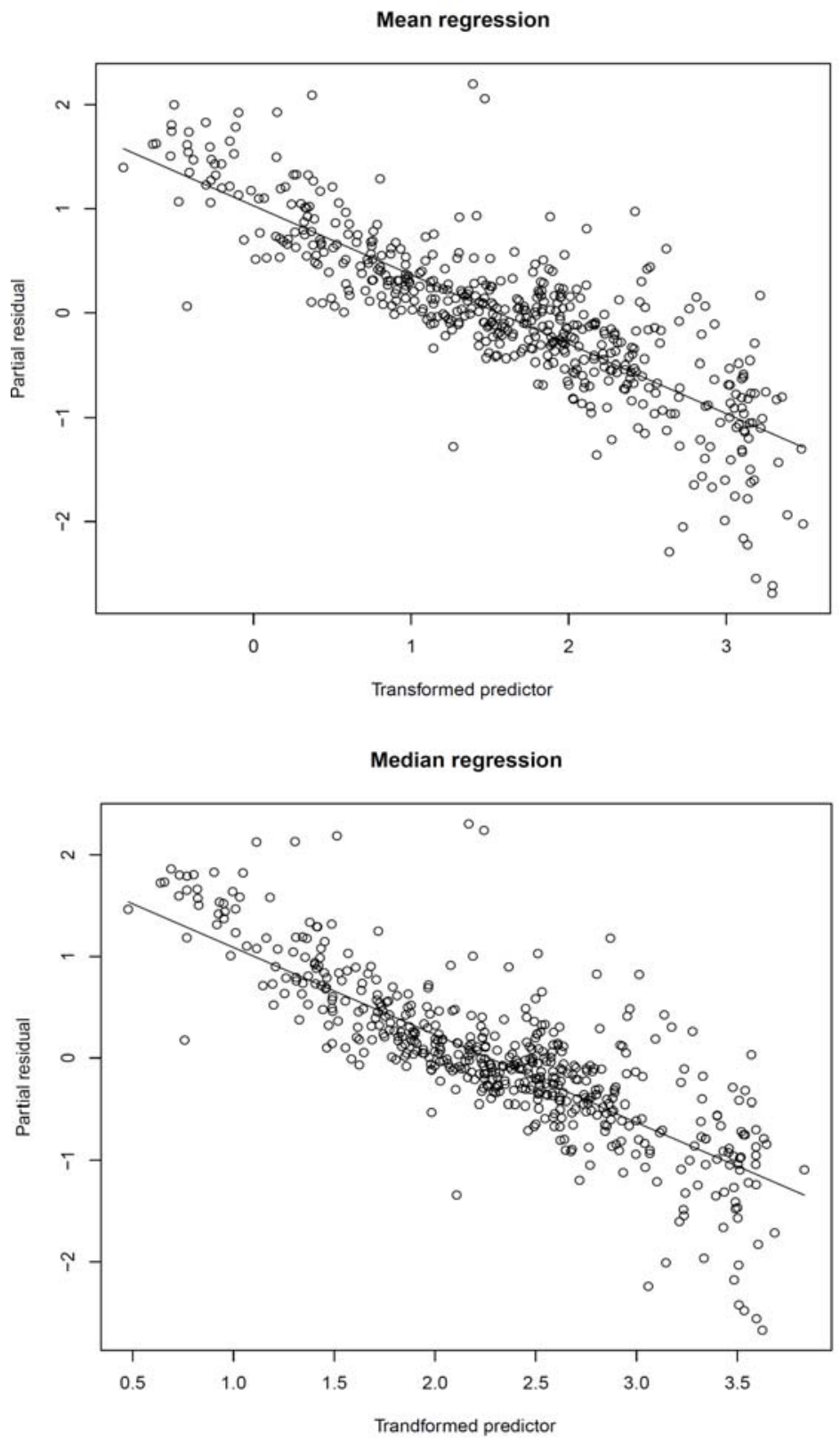

Figure 1. Partial residual $y_{i}-\boldsymbol{x}^{T} \widetilde{\beta}$ versus $t_{i}=\hat{\theta}_{1}^{T} \phi\left(\boldsymbol{z}_{i}\right)$. Left and right panels are for mean regression and median regression. The solid line in each panel is the linear estimator given by $\hat{g}\left(t_{i}\right)=\hat{\mu}_{0}+\hat{w}_{1} t_{i}$. 
Figure 2 shows the contour plot of the estimator of mean surface $g\left(z_{1}, z_{2}\right)$. The left and right panels are for the proposed estimator $\hat{g}(\boldsymbol{z})=\hat{\mu}+\hat{\boldsymbol{w}}^{T} \hat{\Theta} \phi(\boldsymbol{z})$ and the initial estimator $\tilde{g}(\boldsymbol{z})=\tilde{\mu}+\widetilde{u}^{T} \phi(\boldsymbol{z})$, respectively. Roughly speaking, the surfaces were drawn like a plane from the contour line. It seems that the proposed estimator has a smooth surface rather than the initial estimator. In Figure 3, the contour plots of the estimators of median surface are described. In also median regression, the proposed estimator behaved smoothly rather than the estimator without SIR. Thus, the proposed method leads to obtain the estimator with smoothness. 

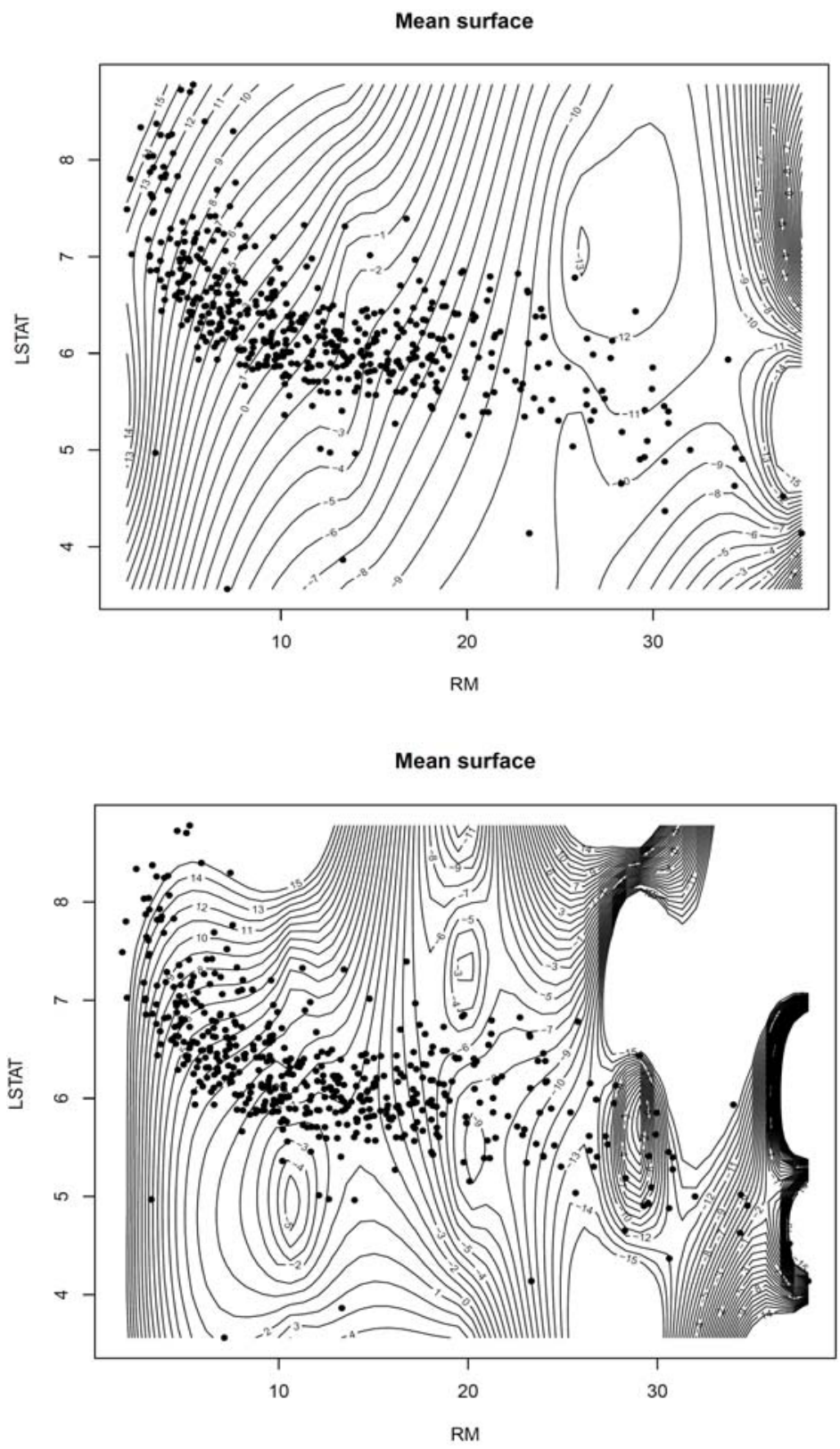

Figure 2. Mean surface estimator of $g\left(z_{1}, z_{2}\right)$. The proposed estimator and initial the estimator are shown in left and right panel, respectively. The dots are location of data points $\boldsymbol{z}_{1}, \cdots, \boldsymbol{z}_{n}$. 

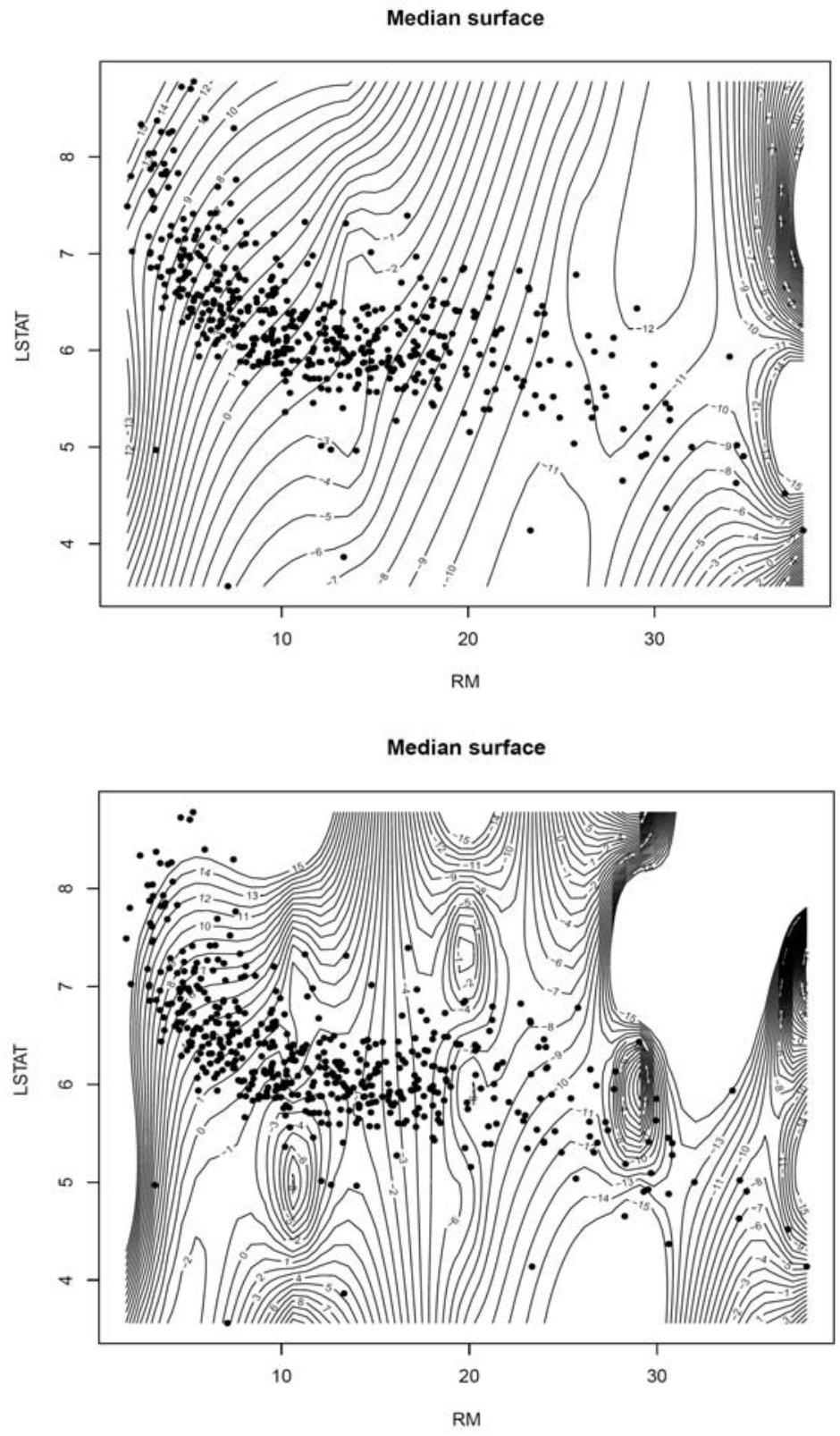

Figure 3. Median surface estimator of $g\left(z_{1}, z_{2}\right)$. The proposed estimator and initial the estimator are shown in left and right panel, respectively. The dots are location of data points $z_{1}, \cdots, z_{n}$. 
From last step of the proposed estimation, we selected the important variable to explain the response by SCAD and Adaptive Lasso. In Table 5 , the estimator of $\beta$ with ordinary method, SCAD, Adaptive Lasso are shown. Here, the ordinary method is the estimation method without penalty. The smoothing parameter included in the penalty was chosen by 5 -fold CV. In mean regression, SCAD selected $x_{2}$ as needless predictor and Adaptive Lasso showed that $x_{2}$ and $x_{3}$ do not affect to the response. The estimator of non zero coefficients were similar for all of ordinary method, SCAD and Adaptive Lasso. In median regression, SCAD and Adaptive Lasso picked $\left\{x_{2}, x_{3}, x_{6}, x_{8}\right\}$, and $\left\{x_{2}, x_{3}, x_{6}, x_{8}, x_{9}\right\}$ as unimportant variable, respectively. It seems that the restriction of Adaptive Lasso is somewhat stronger than that of SCAD. Furthermore, the number of important variables for median estimation was smaller than that for mean estimation. Thus, we see that the mean estimator and median estimator have different structure. 
Table 5. Mean and median estimators of $\beta$ in the Boston Housing data. ORD is unpenalized estimator, SCAD is the estimator with SCAD penalty and AL is the estimator with Adaptive Lasso penalty for both mean and median

\begin{tabular}{lccccccccccc}
\hline Method & $\beta_{1}$ & $\beta_{2}$ & $\beta_{3}$ & $\beta_{4}$ & $\beta_{5}$ & $\beta_{6}$ & $\beta_{7}$ & $\beta_{8}$ & $\beta_{9}$ & $\beta_{10}$ & $\beta_{11}$ \\
\hline Mean & & & & & & & & & & & \\
ORD & -0.441 & 0.042 & 0.083 & 0.101 & -0.372 & 0.057 & -0.358 & 0.517 & -0.434 & -0.309 & 0.121 \\
SCAD & -0.435 & 0 & 0.031 & 0.099 & -0.337 & 0.016 & -0.354 & 0.468 & -0.373 & -0.308 & 0.113 \\
AL & -0.434 & 0 & 0 & 0.101 & -0.321 & 0.004 & -0.367 & 0.450 & -0.348 & -0.301 & 0.112 \\
\hline Median & & & & & & & & & & & \\
ORD & -0.200 & 0.053 & 0.035 & 0.061 & -0152 & 0.017 & -0.199 & 0.181 & -0.179 & -0.178 & 0.108 \\
SCAD & -0.213 & 0 & 0 & 0.016 & -0.162 & 0 & -0.189 & 0 & -0.004 & -0.189 & 0.004 \\
AL & -0.215 & 0 & 0 & 0.058 & -0.044 & 0 & -0.082 & 0 & 0 & -0.159 & 0.072 \\
\hline
\end{tabular}


Table 6 shows that the prediction error (PE) of the estimators. The $\mathrm{PE}$ was defined as that given in previous section. We calculated the prediction error with randomly 5 -fold validation. The initial estimator (without SDR method) with ordinary method, SCAD and Adaptive Lasso are labelled as INI-ORD, INI-SCAD, and INI-AL. The proposed estimator with ordinary method, SCAD and Adaptive Lasso are denoted by SDRORD, SDR-SCAD, and SDR-AL. Overall, the prediction error of the proposed estimator were smaller than that of the initial estimator. For both mean and median regression, SDR-AL had a best prediction error. However, the difference between three estimator with SDR method was quite small.

Table 6. Prediction error of each estimator for both mean and median regression. The labels ORD, SCAD, and $\mathrm{AL}$ are for ordinary method, no, using non penalty, SCAD and Adaptive Lasso penalty, similar to that given in Table 5

\begin{tabular}{lcccccc}
\hline & INI-ORD & INI-SCAD & INI-AL & SDR-ORD & SDR-SCAD & SDR-AL \\
\hline Mean & 0.477 & 0.476 & 0.473 & 0.318 & 0.319 & 0.313 \\
Median & 0.486 & 0.488 & 0.486 & 0.336 & 0.336 & 0.335 \\
\hline
\end{tabular}

Our proposed method is based on the one-step estimation and has non iteration anymore. Even if Steps 2-4 of Section 3 is iterated, the results will be similar. In Figure 4, the partial residuals with one-step iterated $y_{i}-\boldsymbol{x}_{i}^{T} \hat{\beta}$ versus the partial residual with initial $y_{i}-\boldsymbol{x}_{i}^{T} \widetilde{\beta}$ are plotted. All panels are for median regression. In left, middle and right panels, $\hat{\beta}$ is constructed via ordinary method, SCAD and Adaptive Lasso. We see that the partial residuals are almost similar in all methods. Thus, it is sufficient to practice an one-iteration. Similar results were obtained in mean regression. 
TAKUMA YOSHIDA
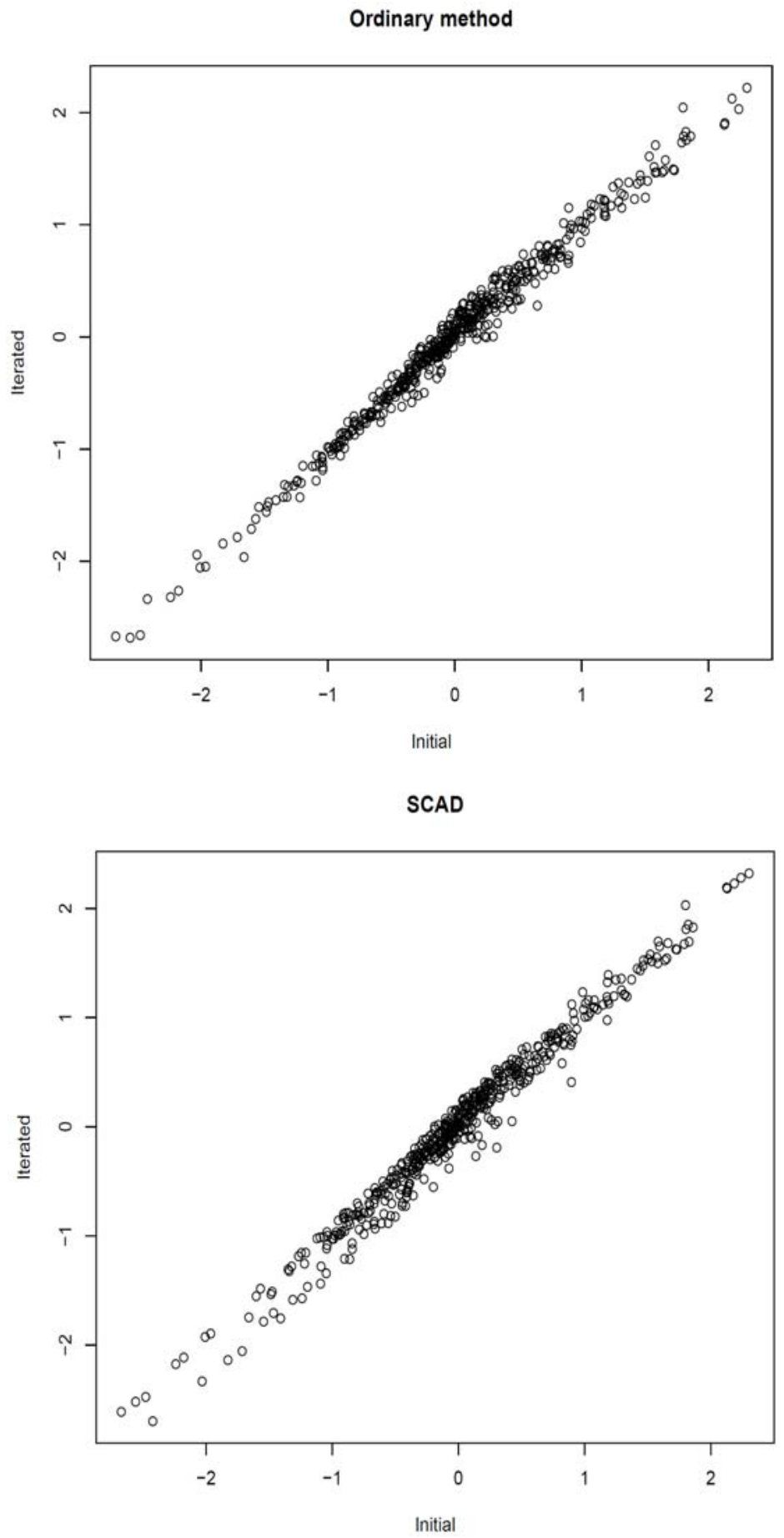


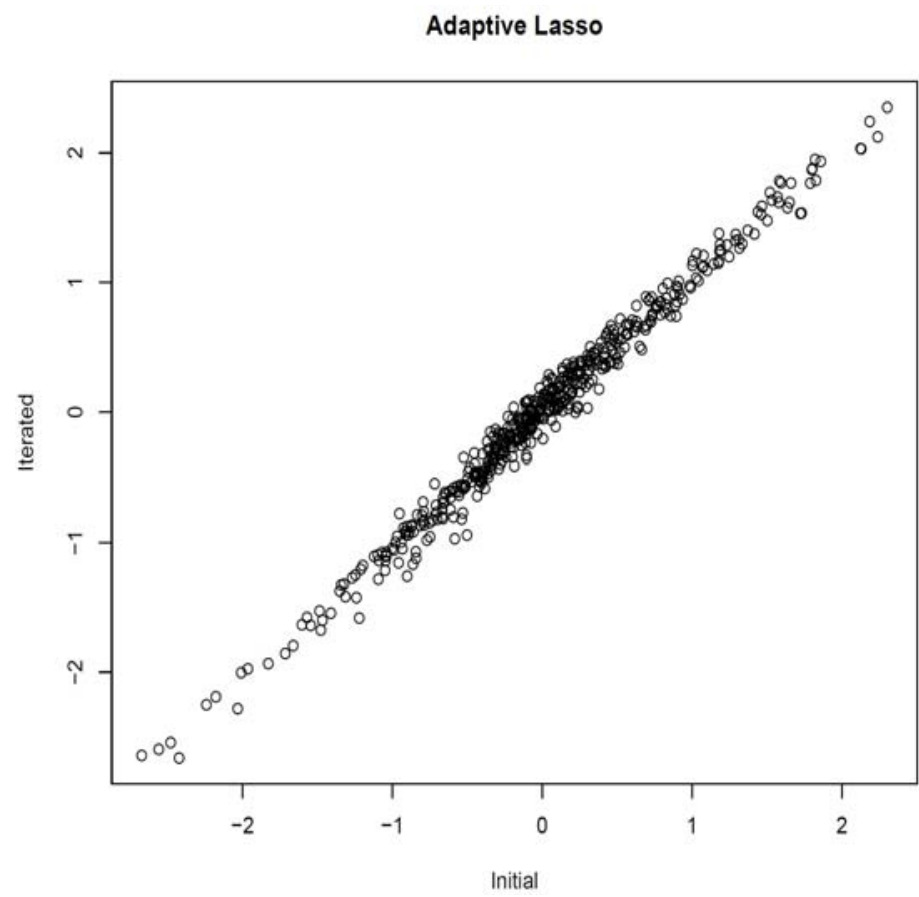

Figure 4. Scatter plot of partial residual with one-step iterated $y_{i}-\boldsymbol{x}_{i}^{T} \hat{\beta}$ versus partial residual with initial $y_{i}-\boldsymbol{x}_{i}^{T} \widetilde{\beta}$ in median regression. The $\hat{\beta}$ of left, middle and right panels are obtained by ordinary, SCAD and Adaptive Lasso, respectively.

\section{Conclusion}

In this paper, we studied the partial linear regression. The variable selection of the parametric component was proceeded by using SCAD and Adaptive Lasso. For the nonlinear component, we use the thin plate spline model. Since the estimates of radial basis function estimator tends to have a wiggly curve, we propose the estimator with smooth curve. The esitmator is the over fitted curve means that its variance is too large. From this, we propose the variance reduction estimator. First the sufficient dimension reduction (SDR) methods to radial basis functions 
are applied for trying to find small number of linear combinations of the radial bases without loss of information. Next, a multiple linear regression model between a response and the transformed radial bases is assumed and estimate the parameters by ordinary $M$-estimation. The estimation is organized as the alternating with one-step iteration. We derived the asymptotic normality of the estimator of coefficients included radial basis function model and the oracle property of the estimator of parametric component. Furthermore, we showed that the estimator with the SDR method has a small variance rather than the ordinary estimator. By simulation and real data application study, the efficiency and the behaviour of the proposed method could be confirmed.

We expect that the proposed method is useful in also binary, count, censored, missing data and so on. As future study, the application to the above data is interesting.

\section{Acknowledgement}

The authors are grateful to Editor, Associate Editor and two anonymous referees for their valuable comments and suggestions, which led to improvements of the paper. The research of the author was partially supported by KAKENHI 26730019.

\section{References}

[1] A. Belloni and V. Chernozhukov, $L_{1}$-penalized quantile regression in highdimensional sparse models, Ann. Statist. 39 (2011), 82-130.

[2] P. K. Bhattacharya and P. L. Zhao, Semiparametric inference in a partial linear model, Ann. Statist. 25 (1997), 244-262.

[3] K. Bollaerts, P. H. C. Eilers and A. Marc, Quantile regression with monotonicity restrictions using $P$-splines and $L_{1}$-norm, Statistical Modelling 6 (2006), 189-207.

[4] R. J. Carroll, J. Fan, I. Gijbels and M. P. Wand, Generalized partially linear singleindex models, J. Amer. Statist. Assoc. 92 (1997), 477-489.

[5] C. H. Chen and K. C. Li, Can SIR be as popular as multiple linear regression, Statist. Sinica 8 (1998), 289-316. 
[6] H. Chen, Convergence rates for parametric components in a partly linear model, Ann. Statist. 16 (1988), 136-141.

[7] X. Chen, C. Zou and R. D. Kook, Coordinate-independent sparse sufficient dimension reduction and variable selection, Ann. Statist. 38 (2010), 3696-3723.

[8] R. D. Cook and F. Critchley, Identifying regression outliers and mixtures graphically, J. Amer. Statist. Assoc. 95 (2000), 781-794.

[9] R. D. Cook and S. Weisberg, Discussion of 'sliced inverse regression for dimension reduction by K. C. Li, J. Amer. Statist. Assoc. 86 (1991), 328-332.

[10] R. D. Cook and L. Ni, Sufficient dimension reduction via inverse regression: A minimum discrepancy approach, J. Amer. Statist. Assoc. 100 (2005), 410-428.

[11] P. Diaconis and D. Freedman, Asymptotics of graphical projection pursuit, Ann. Statist. 12 (1984), 793-815.

[12] K. Doksum and J. Y. Koo, On spline estimators and prediction intervals in nonparametric regression, Comput. Statist. \& Data Anal. 35 (2000), 67-82.

[13] J. Fan and R. Li, Variable selection via nonconcave penalized likelihood and its oracle properties, J. Amer. Statist. Assoc. 96 (2001), 1348-1360.

[14] I. E. Frank and J. H. Friedman, A statistical view of some chemometrics regression tools (with discussion), Technometrics 35 (1993), 109-148.

[15] W. Härdle, Hua Liang and J. Gao, Partially Linear Models, Springer, New York, 2000 .

[16] D. Harrison and D. Rubinfeld, Hedonic housing pries and the demand for clean air, Journal of Environmental Economics and Management 5 (1978), 81-102.

[17] X. He and B. Shi, Bivariate tensor-product $B$-splines in a partially linear regression, J. Multivariate Anal. 58 (1996), 162-181.

[18] N. Heckman, Spline smoothing in a partly linear model, J. Roy. Statist. Soc. Ser. A 48 (1986), 244-248.

[19] T. Hoshino, Quantile regression estimation of partially linear additive models, Journal of Nonparametric Statistics 26 (2014), 509-536.

[20] W. Jiang, H. D. Bondell and H. J. Wang, Interquantile shrinkage and variable selection in quantile regression, Comput. Statist. \& Data Anal. 69 (2014), 208-219.

[21] K. Knight, Limiting distributions for $L_{1}$ regression estimators under general conditions, Ann. Statist. 26 (1998), 755-770.

[22] R. Koenker and G. Bassett, Regression quantiles, Econometrica 46 (1978), 33-50.

[23] R. Koenker and N. S. Portnoy, Quantile smoothing splines, Biometrika 81 (1994), 673-680.

[24] S. Lee, Efficient semiparametric estimation of a partially linear quantile regression model, Econometric Theory 19 (2003), 1-31. 
[25] K. C. Li, Sliced inverse regression for dimension reduction, J. Amer. Statist. Assoc. 102 (1991), 997-1008.

[26] K. C. Li, On principal Hessian directions for data visualization and dimension reduction: Another application of Stein's lemma, J. Amer. Statist. Assoc. 87 (1992), 1025-1039.

[27] L. Li, Sparse sufficient dimension reduction, Biometrika 94 (2007), 603-613.

[28] B. Li and S. Wang, On directional regression for dimension reduction, J. Amer. Statist. Assoc. 33 (2007), 1580-1616.

[29] Y. Li and L. X. Zhu, Asymptotics for sliced average variance estimation, Ann. Statist. 35 (2007), 41-69.

[30] Y. Li and L. J. Zhu, $L_{1}$-norm quantile regression, J. Comput. Graph. Statist. 17 (2008), 163-185.

[31] X. Liu, L. Wang and H. Liang, Estimation and variable selection for semiparametric additive partial linear models, Stat. Sin. 21 (2011), 1225-1248.

[32] Y. Ma and L. Zhu, On estimation efficiency of the central mean subspace, J. Roy. Statist. Soc. B 76 (2014), 885-901.

[33] D. Pollard, Asymptotics for least absolute deviation regression estimators, Econometric Theory 7 (1991), 186-199.

[34] P. T. Reiss and L. Huang, Smoothness selection for penalized quantile regression splines, The International Journal of Biostatistics (2012), 8-1.

[35] D. Ruppert, M. P. Wand and R. J. Carroll, Semiparametric Regression, Cambridge Univ. Press, New York, 2003.

[36] Y. Sun, Semiparametric efficient estimation of partially linear quantile regression models, Annals of Economics and Finance 6 (2005), 105-127.

[37] R. Tibshirani, Regression shrinkage and selection via the lasso, J. Roy. Statist. Soc. Ser. B 58 (1996), 267-288.

[38] D. Tyler, Asymptotic inference for eigenvectors, Ann. Stat. 9 (1981), 725-736.

[39] S. N. Wood, Thin plate regression splines, J. R. Statist. Soc. B 65 (2003), 95-114.

[40] Y. Wu and Y. Liu, Variable selection in quantile regression, Statistica Sinica 19 (2009), 801-817.

[41] Y. Xia and W. Härdle, Semi-parametric estimation of partially linear single-index models, J. Multivariate Anal. 97 (2006), 1162-1184.

[42] Y. Xia, H. Tong, W. K. Li and L. X. Zhu, An adaptive estimation of dimension reduction space, J. Roy. Statist. Soc. Ser. B 64 (2002), 363-410.

[43] Luo Xiao, Y. Li and D. Ruppert, Fast bivariate $P$-splines: The Sandwich smoother, J. Roy. Statist. Soc. B 75 (2013), 577-599.

[44] Z. Ye and R. E. Weiss, Using the bootstrap to select one of a new class of dimension reduction methods, J. Amer. Statist. Assoc. 98 (2003), 968-979. 
[45] P. Yoo and R. D. Cook, Optimal sufficient dimension reduction for the conditional mean in multivariate regressions, Biometrika 94 (2007), 231-242.

[46] T. Yoshida, Partially linear estimation using sufficient dimension reduction, Submitted (2015).

[47] L. X. Zhu and K. W. Ng, Asymptotics of sliced inverse regression, Statist. Sinica 5 (1995), 727-736.

[48] L. X. Zhu, B. Q. Miao and H. Peng, Sliced inverse regression with large-dimensional covariates, J. Amer. Statist. Assoc. 101 (2006), 630-643.

[49] L. P. Zhu, R. Li and H. Cui, Robust estimation for partially linear models with largedimensional covariates, Science China Mathematics 56 (2013), 2069-2088.

[50] H. Zou, The adaptive lasso and its oracle properties, J. Amer. Statist. Assoc. 101 (2006), 1418-1429.

\section{Appendix}

The theorems in this paper can be proven by existing results. The Theorem 1 can be shown by applying the convexity lemma of Pollard [33]. The proofs of Theorems 2 and 3 are almost similar to that of Theorems 2 and 3 of $\mathrm{Wu}$ and Liu [40], which was studied the variable selection of linear quantile regression. Thus, by combining the existing result, the asymptotic result of the proposed method can be evaluated. However, we briefly mention the outline of its proofs.

Proof of Lemma 1. Let $\boldsymbol{V}_{i}=\left[1, \boldsymbol{X}_{i}^{T}, \phi\left(\boldsymbol{Z}_{i}\right)^{T}\right]^{T}$ and $c=\left[\mu, \beta^{T}, \boldsymbol{u}^{T}\right]^{T}$. Then the model can be written as $f\left(\boldsymbol{x}_{i}, \boldsymbol{z}_{i}\right)=v_{i}^{T} \boldsymbol{c}_{0}$, where $\boldsymbol{c}_{0}=\left[\mu_{0, \text { ini }}\right.$, $\left.\beta_{0, \text { ini }}^{T}, \boldsymbol{u}_{0}^{T}\right]^{T}$. Let $U_{i}=Y_{i}-v_{i}^{T} \boldsymbol{c}_{0}$ and let

$$
Q(\delta)=\sum_{i=1}^{n} \rho\left(U_{i}-\delta^{T} v_{i} / \sqrt{n}\right)-\rho\left(U_{i}\right) .
$$

Then the minimizer $\widetilde{\delta}$ of $Q(\delta)$ is

$$
\widetilde{\delta}=\sqrt{n}\left(\tilde{\boldsymbol{c}}-\boldsymbol{c}_{0}\right),
$$

where $\tilde{\boldsymbol{c}}=\left[\tilde{\mu}, \widetilde{\beta}^{T}, \tilde{\boldsymbol{u}}^{T}\right]^{T}$. We now define 


$$
R(\delta)=Q(\delta)-E[Q(\delta)]-\sum_{i=1}^{n}\left\{\rho^{\prime}\left(U_{i}\right)-E\left[\rho^{\prime}\left(U_{i}\right)\right]\right\}\left(n^{-1 / 2} \delta^{T} v_{i}\right)
$$

We note that $E[Q(\delta)]$ is continuous with respect to $\delta \in \mathbb{R}^{1+p+K}$ from Assumption 1(a). The expectation of $R(\delta)$ is 0 and tedious but simple calculation yields that $V[R(\delta)]=o(1)$ by Assumption 1(a) and (b). Thus, we have

$$
\begin{aligned}
Q(\delta) & =E[Q(\delta)]+\sum_{i=1}^{n}\left\{\rho^{\prime}\left(U_{i}\right)-E\left[\rho^{\prime}\left(U_{i}\right)\right]\right\}\left(n^{-1 / 2} \delta^{T} v_{i}\right)+o_{P}(1) \\
& =E[Q(\delta)]-\sum_{i=1}^{n} E\left[\rho^{\prime}\left(U_{i}\right)\right] n^{1 / 2} \delta^{T} v_{i}+n^{-1 / 2} \delta^{T} v_{i} \sum_{i=1}^{n} \rho^{\prime}\left(U_{i}\right)+o_{P}(1) .
\end{aligned}
$$

Again from Assumption 1(a), we use Taylor expansion around $\delta^{T} v_{i} / \sqrt{n}=0$ and obtain

$$
\begin{aligned}
E[Q(\delta)]-\sum_{i=1}^{n} E\left[\rho^{\prime}\left(U_{i}\right)\right] n^{-1 / 2} \delta^{T} v_{i}= & \frac{1}{2} \delta^{T}\left(\frac{1}{n} \sum_{i=1}^{n} E\left[\rho^{\prime \prime}\left(U_{i}\right)\right] v_{i} v^{T}\right) \delta+o(1) \\
& \stackrel{P}{\rightarrow} \frac{1}{2} \delta^{T} C\left(\left\{\rho^{\prime \prime}\right\}\right) \delta
\end{aligned}
$$

as $n \rightarrow \infty$. Next Assumption 1(c) guarantees that the second term of right hand side of (9) satisfies the Lyapunov condition for central limit theorem. Therefore we have as $n \rightarrow \infty$,

$$
n^{-1 / 2} \delta^{T} v_{i} \sum_{i=1}^{n} \rho^{\prime}\left(U_{i}\right) \stackrel{D}{\rightarrow} \delta^{T} \boldsymbol{W}
$$

where $\boldsymbol{W} \sim N\left(\mathbf{0}, C\left(\left\{\rho^{\prime}\right\}^{2}\right)\right)$. Consequently, as $n \rightarrow \infty$,

$$
Q(\delta) \stackrel{D}{\rightarrow} Q_{0}(\delta) \equiv \frac{1}{2} \delta^{T} C\left(\left\{\rho^{\prime \prime}\right\}\right) \delta+\delta^{T} W
$$


From convexity lemma of Pollard [33] yields that the minimizer of $Q$ converges to the minimizer of $Q_{0}$, and hence

$$
\widetilde{\delta} \stackrel{D}{\rightarrow} C\left(\left\{\rho^{\prime \prime}\right\}\right)^{-1} \mathbf{W}
$$

can be obtained. This completes the proof.

Proof of Lemma 2. Although the proof of Lemma 2 is similar to that of Theorem 2 of Zhu and $\mathrm{Ng}$ [47] and $\mathrm{Li}$ and $\mathrm{Zhu}$ [29], we note Assumption 2(c) concerned with the difference between $\widetilde{\beta}$ and $\beta_{0}$. Let

$$
\begin{aligned}
& M_{S I R}=\operatorname{Cov}\left(E\left[\phi^{*}(\boldsymbol{Z}) \mid y-\boldsymbol{x}^{T} \beta_{0}\right]\right), \\
& \tilde{M}_{S I R}=\operatorname{Cov}\left(E\left[\phi^{*}(\boldsymbol{Z}) \mid y-\boldsymbol{x}^{T} \widetilde{\beta}\right]\right), \\
& M_{S A V E}=G^{1 / 2} E\left[\left\{I-\operatorname{Cov}\left(\phi^{*}(\boldsymbol{Z}) \mid y-\boldsymbol{x}^{T} \beta_{0}\right)\right\}^{2}\right] G^{1 / 2}, \\
& \tilde{M}_{S A V E}=G^{1 / 2} E\left[\left\{I-\operatorname{Cov}\left(\phi^{*}(\boldsymbol{Z}) \mid y-\boldsymbol{x}^{T} \widetilde{\beta}\right)\right\}^{2}\right] G^{1 / 2} .
\end{aligned}
$$

From Assumption 2(c), we have

$$
\begin{aligned}
& m\left(y-\boldsymbol{x}^{T} \widetilde{\beta}\right)=m\left(y-\boldsymbol{x}^{T} \beta_{0}\right)+O_{P}\left(n^{-1 / 2}\right), \\
& M\left(y-\boldsymbol{x}^{T} \widetilde{\beta}\right)=M\left(y-\boldsymbol{x}^{T} \beta_{0}\right)+O_{P}\left(n^{-1 / 2}\right),
\end{aligned}
$$

and straightforward calculation yields $\tilde{M}_{S I R}=M_{S I R}+O_{P}\left(n^{-1 / 2}\right)$ and $\tilde{M}_{S A V E}=M_{S A V E}+O_{P}\left(n^{-1 / 2}\right)$. Direct use of Theorem 2 of Zhu and Ng [47] and Theorems 2.1 and 2.2 of $\mathrm{Li}$ and $\mathrm{Zhu}$ [29] lead to

$$
\sqrt{n}\left(\hat{M}_{S I R}-\tilde{M}_{S I R}\right)=O_{P}(1), n^{\beta}\left(\hat{M}_{S A V E}-\tilde{M}_{S A V E}\right)=O_{P}(1),
$$

for $0<\beta<1 / 2$. Thus, for SIR,

$$
\sqrt{n}\left(\hat{M}_{S I R}-M_{S I R}\right)=\sqrt{n}\left(\hat{M}_{S I R}-\tilde{M}_{S I R}+\tilde{M}_{S I R}=M_{S I R}\right)=O_{P}(1)
$$


Similarly, we have $n^{\beta}\left(\hat{M}_{S A V E}-M_{S A V E}\right)=O_{P}(1)$. From the perturbation theory (for example; Tyler [38]), $\hat{\theta}_{j}(j=1, \cdots, d)$, which is the eigenvectors of $\hat{G}^{-1} \hat{M}$ converge to the corresponding eigenvectors of $G^{-1} M$.

Outline of proof of Theorem 1. The proof of Theorem 1 is similar to that of Lemma 1 . We replace $U_{i}, \delta$ in the proof of Lemma 1 to $\widetilde{U}_{i}=Y_{i}-\mu_{0}-\boldsymbol{X}_{i}^{T} \widetilde{\beta}-\boldsymbol{w}_{0}^{T} \hat{\Theta} \phi\left(\boldsymbol{Z}_{i}\right)$ and $v_{i}=\left[1,\left(\Theta_{0} \phi\left(\boldsymbol{z}_{i}\right)\right)^{T}\right]^{T}$. Then we have $\tilde{U}_{i}=U_{i}+o_{P}(1)$. Therefore, the Theorem 1 can be shown in the same manner as Lemma 1.

Outline of proof of Theorems 2 and 3 . The oracle property of the parametric estimator is generally shown by convexity lemma similar to Theorem 1. Since the penalty term of SCAD and Adaptive Lasso do not contain the information of loss function $\rho$, the convergence function of loss function $\rho$ plus penalty can be considered as the convergence function of the loss function plus the convergence function of the penalty. The convergence function of loss function have already been studied by Theorem 1. The asymptotic form of the penalty for SCAD and Adaptive Lasso were given by $\mathrm{Wu}$ and $\mathrm{Liu}$ [40]. In $\mathrm{Wu}$ and Liu [40], by replacing the check function with $\rho$, the proofs of Theorems 2 and 3 of $\mathrm{Wu}$ and Liu [40] can directly be applied in our problem. Thus, Theorems 2 and 3 are shown. 